\title{
Electrical Transport in Crystalline and Amorphous Chalcogenide
}

Daniele lelmini

Dipartimento di Elettronica, Informazione e Bioingegneria

Politecnico di Milano

Piazza L. da Vinci 32

20133 Milano (Italy)

Email: daniele.ielmini@polimi.it 


\section{Table of Contents}

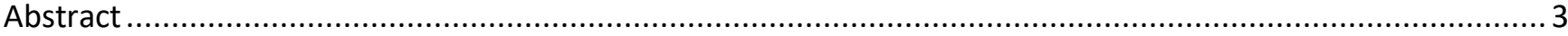

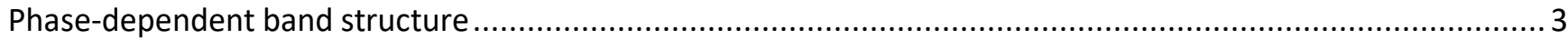

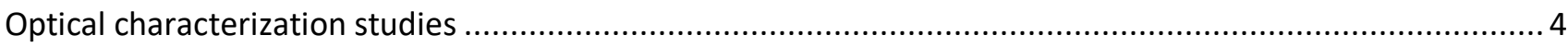

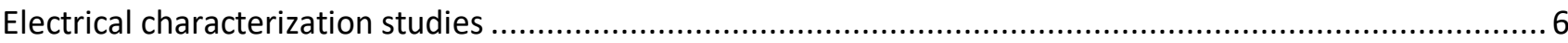

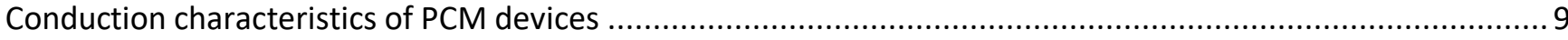

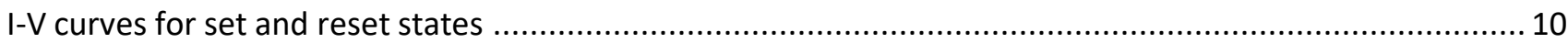

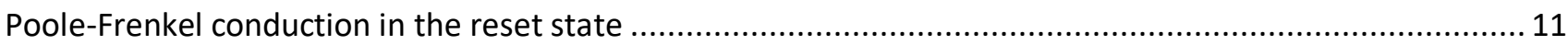

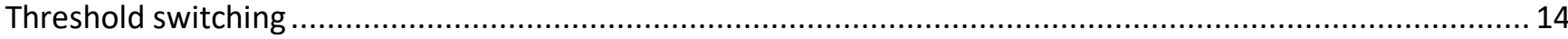

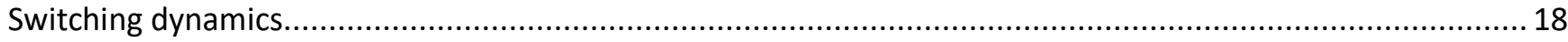

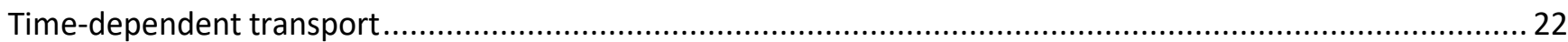

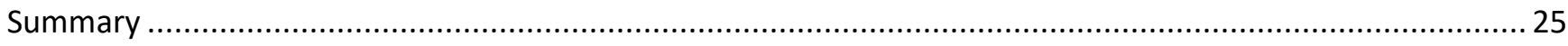

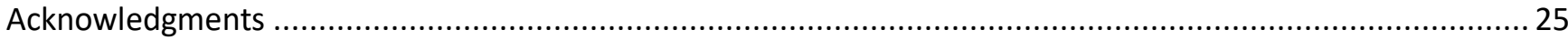

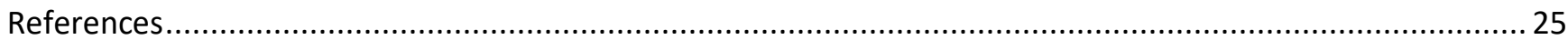




\section{Abstract}

Phase change memory (PCM) operation relies on a combination of electrical, thermal and structural effects at the nanoscale [1-5]. Under an externally-applied electric field, electrical current flows across the device and cause power dissipation and a consequent increase of local temperature, thus inducing crystallization or melting/amorphization, depending on temperature (hence on voltage and current). The corresponding change of the device resistance can then be sensed by application of a relatively low voltage. Electrical characteristics of the crystalline and amorphous phases thus play a fundamental role in the programming and read behavior of the PCM device. The electrical current-voltage characteristics of the PCM device, for instance, control the read current margin, the programming current, and the energy consumption during memory array operation. To design the driving circuits for generating the voltage pulses for memory program and read, or for selecting the correct select device to allow selection/unselection of memory bits within a crosspoint array, the device characteristics are an essential piece of information. The same applies to sensing circuits aimed at reading the memory state. Finally, the dependence of voltage and currents on the device geometry (e.g., thickness of the active layer, diameter of the bottom electrode) plays a leading role in determining the scaling behavior of the PCM device for various technology nodes. Optimization of PCM device should include a careful consideration of the electrical behavior, in combination to structural properties (melting and crystallization point, activation energy of crystallization, etc.) and thermal characteristics (thermal conductivity, thermal boundary resistances, etc.).

This chapter summarizes the fundamental electrical properties of PCM devices in both the amorphous and crystalline states. First, the band structure of crystalline and amorphous phase change materials will be studied based on the analysis of thin films of active materials. Then, the device characteristics in a PCM device including conduction and threshold switching phenomena will be shown. Finally, the effects of non-uniform resistivity, the transient phenomena after switching, and the thermo-electric effects due to the coupling of the temperature distribution with the electrical characteristics will be illustrated.

Keywords: phase change memory, threshold switching, Poole-Frenkel transport, band structure, localized states, energy landscape, chalcogenide materials, structural relaxation, resistance drift, multilevel memory.

\section{Phase-dependent band structure}

Thin films represent a valuable vehicle for characterizing the electrical properties of materials, e.g., the electrical resistivity in different phases and temperature, as well as for carrying out optical investigations (e.g., by photon absorption or reflection) of the band structure for various structural phases. Some properties of the band structure, e.g., the energy gap, its direct/indirect character, the presence of tails of localized states, etc., can in fact be characterized almost directly from optical experiments, thus providing a key input into the physical mechanisms for conduction that are responsible for the electrical properties of the materials. 
Most of the results shown in the following refer to $\mathrm{Ge}_{2} \mathrm{Sb}_{2} \mathrm{Te}_{5}$ (GST), which is by far the most widely studied phase change material for optical and electrical storage since the seminal work of Yamada et al. [6]. In fact, GST features a unique combination of excellent properties, such as thermodynamic stability, within the Ge-Sb-Te ternary phase diagram, practical melting temperature $\left(T_{m}=620^{\circ} \mathrm{C}\right.$ ), fast crystallization (in the range of $100 \mathrm{~ns}$ for sufficiently high temperatures), and good glass forming ability, in that the maximum quenching time to achieve an amorphous phase by cooling a liquid phase is in the range of about $50 \mathrm{~ns}$ [7], thus easily achievable within a nanoscale PCM device connected to a memory circuits. For these reasons, GST is the most popular material used in optical data storage, such as rewritable CD and DVD, and in PCM devices [8-10].

(a)

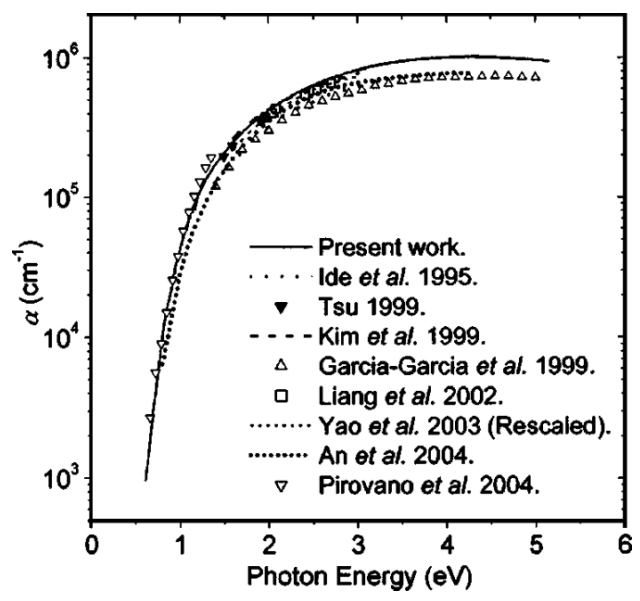

(b)

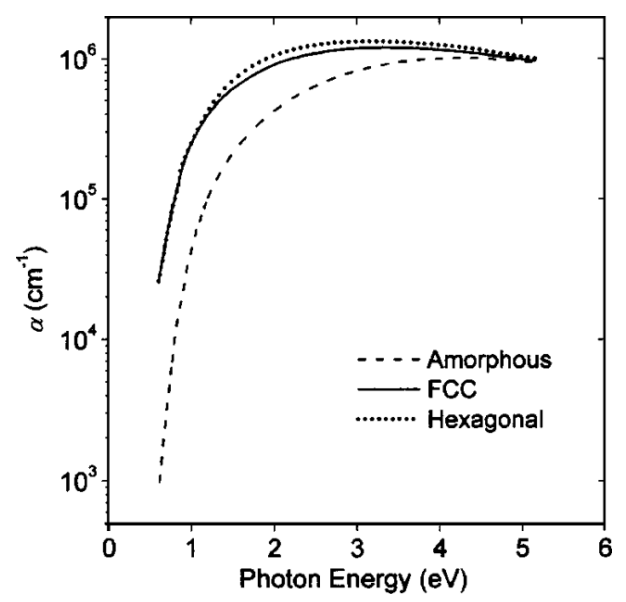

Fig. 1 Measured absorption coefficient a as a function of photon energy for amorphous GST (a) and comparison between crystalline and amorphous GST (b) [11].

\section{Optical characterization studies}

Fig. 1a shows the measured absorption coefficient $\alpha$ of GST in the amorphous phase as a function of photon energy hv [11]. Data in the figure were obtained from ellipsometry analysis or optical transmission/reflection studies and compare results obtained from various authors in the literature [11-19], which all show a substantial agreement. Small differences might be attributed to a slightly different stoichiometric composition and/or degree of film oxidation, resulting from the different techniques of deposition. The steep drop of $\alpha$ for decreasing optical energies indicate the band edge. To carefully determine the optical band gap $E_{g, o p t}$, data were fitted to the Tauc equation $[20,21]$ :

$\alpha h v=C\left(h v-E_{g, o p t}\right)^{r}$,

where $C$ and $r$ are constants. Using an exponent $r=2$, which adequately describes optical electron-hole generation in an amorphous semiconductor where $k$ is not conserved in the transition [21], and extrapolating to the abscissa, a band gap $\mathrm{E}_{\mathrm{g}, \mathrm{opt}}=0.7 \mathrm{eV}$ could be evaluated $[11,19]$. More careful analysis indicates a marked deviation from the slope $r=2$ at low energies below about $0.9 \mathrm{eV}$, which reveal the presence of exponentiallydecaying Urbach tails due to localized states. These states are generally attributed to disorder in the amorphous state, according to the Anderson theory of disorder-induced localization of states in the band gap 
[20]. Such localized states, which are found to belong to a Urbach tail of about $80 \mathrm{meV}$, have a key role in conduction and switching in the amorphous phase. Similar results for the absorption spectrum and band structure were obtained by subsequent studies [22]. Absorption data were also compared with spectral photoconductivity, measuring the electrical current under illumination of monochromatic light at a given photon energy hv, indicating a substantial agreement and confirming the band structure picture for amorphous GST.

Fig. $1 \mathrm{~b}$ shows the comparison of measured $\alpha$ for GST in the crystalline and amorphous phases. Two crystalline phases are considered for GST, namely the metastable face-centered cubic (fcc) structure and the stable hexagonal structure [6]. The measured $\alpha$ shows a negligible difference between the 2 phases, and a substantially larger value for crystalline phases compared to the amorphous state. This can be understood by a shift of the absorption spectrum to lower photon energies due to a smaller energy gap. Linear fitting in the Tauc plot with $r=2$ and extrapolation to zero absorption results in an estimated optical energy gap of $0.5 \mathrm{eV}$ [11], in substantial agreement with other reports $[14,15,17,19]$.

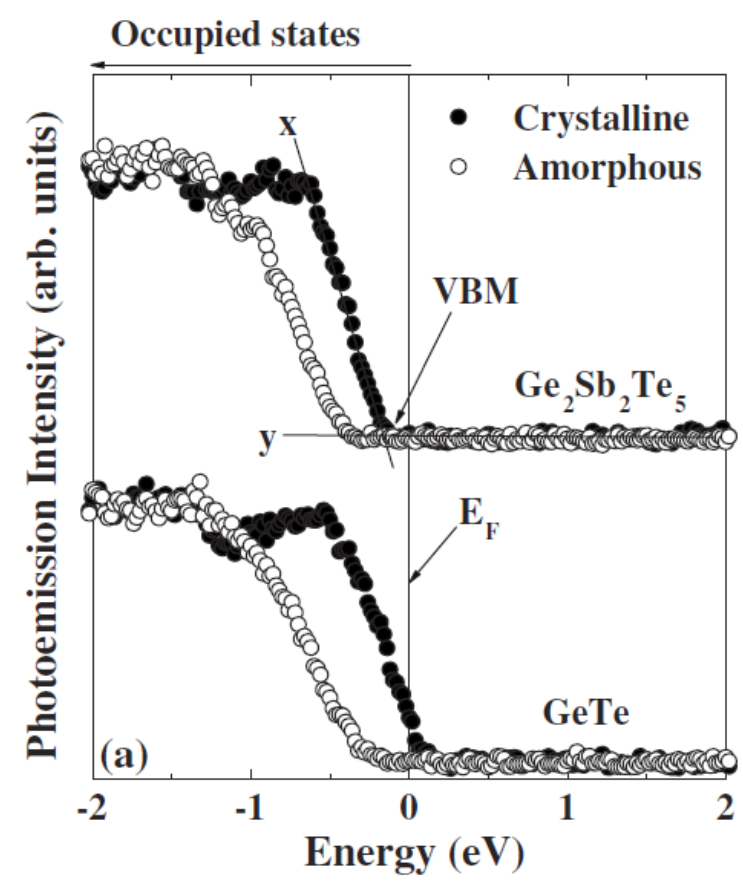

Fig. 2 HXPES spectra for GST (top) and GeTe (bottom) in the amorphous and crystalline phases [23].

Understanding the electrical properties of GST also requires the assessment of the position of the Fermi level, which determines the population of the density of states in the conduction and valence band. To this purpose, Fig. 2 shows the experimental results for the hard x-ray photoemission spectroscopy (HXPES) of GST and GeTe [23]. HXPES is a valuable technique to investigate the valence band structure of electronic materials and allows to extract the density of states in the valence band with respect to the Fermi level. The figure shows the HXPES 
spectra for the amorphous and crystalline phases, as a function of energy, where $E=0$ represent the equilibrium Fermi energy. The valence band edge is generally closer to the Fermi level for the crystalline phase compared to the amorphous phase, supporting the larger conductivity in the crystalline phase which is the basis of the storage concept for PCM devices. For GST, the difference between $E_{F}$ and the valence band edge in the amorphous phase is about $0.4 \mathrm{eV}$, i.e., about half of the band gap, indicating that amorphous GST behaves as an intrinsic semiconductor where the Fermi level is pinned at midgap. In the crystalline (fcc) phase, GST shows a deeper valence band compared to GeTe, where $\mathrm{E}_{\mathrm{F}}$ lies almost in correspondence of the valence band edge, thus suggesting a degenerate doping concentration and almost metallic conduction. Doping of $p$-type character is generally attributed to vacancies, which can reach an atomic concentration of about $20 \%$ in GST due to vacancies of the Ge/Sb sub-lattice $[19,24]$, mostly surrounded by Te neighbors [25].

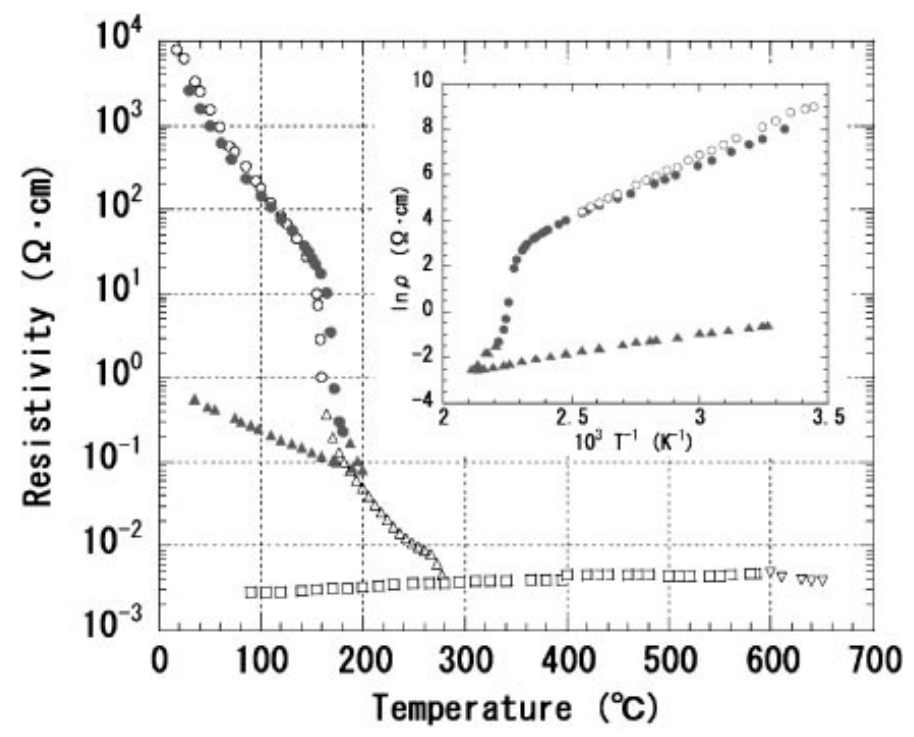

Fig. 3 Measured resistivity as a function of temperature for GST in the amorphous and crystalline phases. The film thickness was $110 \mathrm{~nm}$ (open symbol) and $510 \mathrm{~nm}$ (solid symbol). The inset shows the same data reported in the Arrhenius plot [22].

\section{Electrical characterization studies}

Electrical measurements allow a more direct investigation of carrier transport properties, such as the type of majority carriers and their concentration and mobility. Fig. 3 shows the measured resistivity as a function of temperature for GST in the amorphous and crystalline phase. In these measurements, the as-grown amorphous GST film was characterized at increasing temperature from $20^{\circ} \mathrm{C}$ to $650^{\circ} \mathrm{C}$, thus above the melting temperature. At relatively low temperature below $150^{\circ} \mathrm{C}$, the amorphous resistivity shows a regular decrease, which can be attributed to the temperature activation of transport in the intrinsic amorphous semiconductor, most probably arising from thermal generation of carriers from the valence to the conduction band with activation energy equal to half of the band gap. The activation energy can be estimated from the slope of resistivity in the Arrhenius plot shown in the inset. Inspection of the Arrhenius plot indicates an activation 
energy of about $0.45 \mathrm{eV}$ in close agreement with the difference between $\mathrm{E}_{\mathrm{F}}$ and the valence band edge in Fig. 2. As the temperature reaches about $170^{\circ} \mathrm{C}$, the resistivity shows a marked drop, thus revealing thermallyinduced crystallization to the fcc phase. If the temperature is decreased soon after crystallization, resistivity increases for decreasing temperature but with a smaller slope compared to the amorphous phase. The Arrhenius plot shows an activation energy of about $0.14 \mathrm{eV}$, which may be attributed to the energy difference between $\mathrm{E}_{\mathrm{F}}$ and the valence band edge for fcc crystalline GST in Fig. 2. After the steep region of crystallization, resistivity shows a further decrease with a shallower slope, probably associated to the nucleation and growth of hexagonal grains within the fcc metastable phase. Exceeding the melting temperature does not result in major changes of resistivity, suggesting that the conduction properties of liquid GST are similar to those of the hexagonal phase, which behaves as a degenerate semiconductor, or a metal, given the increase of resistivity with temperature.

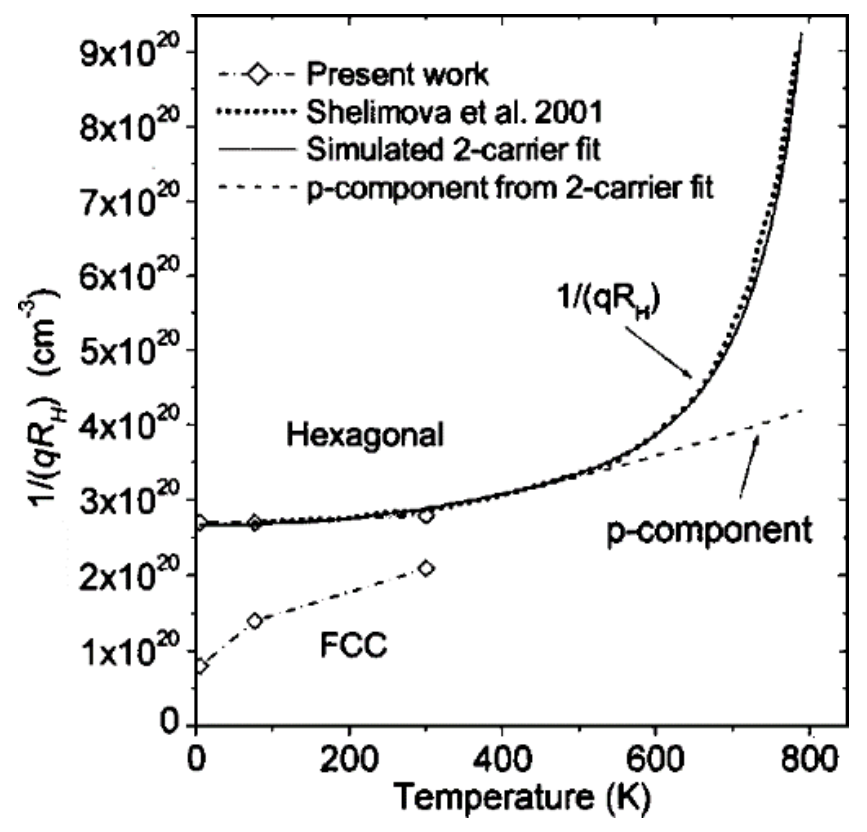

Fig. 4 Measured $1 /\left(\sigma R_{H}\right)$, namely the inverse product of electrical conductivity $\sigma$ and Hall coefficient $R_{H}$, for the hexagonal and the fcc phases of GST [11,26]. Data indicate a p-type doping, consistent with the large concentration of $\mathrm{Ge}$ and $\mathrm{Sb}$ vacancies [22].

Additional insight into the transport properties of amorphous and crystalline GST can be gained by more sophisticated experiments, such as Hall conductivity measurements. Fig. 4 shows the measured $1 /\left(\sigma R_{H}\right)$, namely the inverse product of electrical conductivity $s$ and Hall coefficient $R_{H}$, for the hexagonal and the fcc phases of GST [11,26]. Data indicate a p-type doping, consistent with the large concentration of Ge and $\mathrm{Sb}$ vacancies. An estimate of hole concentration can be derived from the inverse product $1 /\left(\sigma R_{H}\right)$, which is in the range of few $10^{20} \mathrm{~cm}^{-3}$ at room temperature. A larger hole concentration is found for the hexagonal phase compared to the fcc phase, in agreement with the lower resistivity in Fig. 3. Hall measurements are generally 
extremely difficult for the amorphous phase, due to the extremely low current. However, a p-type character of conduction was assessed for the amorphous phase via Seeback analysis [22]. This supports the picture of slightly p-type amorphous GST, although its main character remains that of an intrinsic material with very poor concentration of carriers in the extended states. This remains the main origin of the resistivity ratio and optical contrast at the basis of electrical and optical phase change memories, respectively.

Fig. 5 shows the schematic band structure for the crystalline (top) and the amorphous phases (bottom) of GST [27]. The crystalline phase is a degenerate semiconductor with relatively narrow band gap, where the Fermi lies close to the valence band maximum due to vacancy-induced self-doping. The amorphous phase appears as an intrinsic semiconductor with the Fermi level close to midgap, as a result of defect-induced pinning. Defects may consist of distorted bonds, wrong bonds (e.g., Ge-Ge bonds in GST) and dangling bonds, which arise due to the disordered structure. Defects also provide localized states in the forbidden gap for capture and emission of carries to/from the band of extended states [28-30]. Although these localized states are preferentially located close to the band edges in the form of Urbach tails, a finite amount of localized state density might take place at the Fermi level deep in the band gap. The different position of the Fermi level eventually accounts for the different electrical resistivity in the 2 phases at the origin of the PCM storage concept.

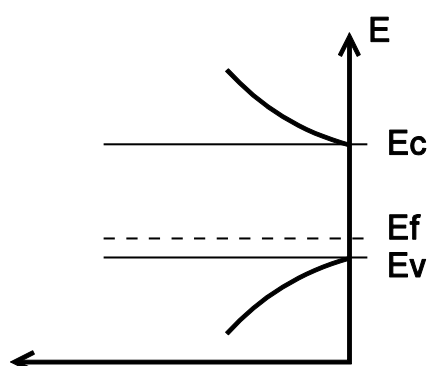

$N(E)$

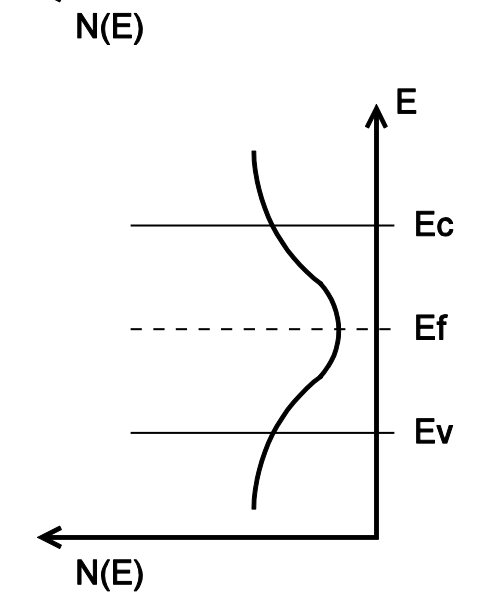

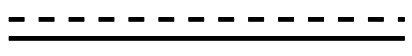

(a) Crystalline phase

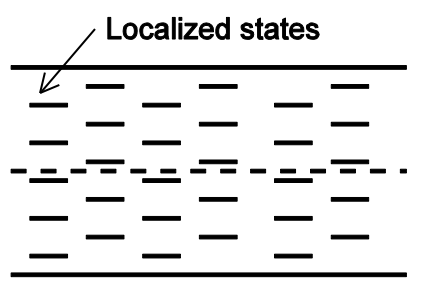

(b) Amorphous phase

Fig. 5 Band structure of the crystalline (top) and amorphous phases (b) of GST [27]. 


\section{Conduction characteristics of PCM devices}

While thin films remain the preferred vehicle for basic understanding and exploration of novel materials, the PCM device characteristics if the source of the main information regarding the device and its electrical parameters. For instance, important device properties such as the resistivity of the amorphous phase obtained by quenching from the melt and the threshold switching voltage cannot be obtained from a planar thin film analysis due to the limited electric field and temperature in these experiments.

A PCM device generally consists of a layer of phase-change material, such as GST, between 2 electrical contacts. Fig. 6 shows the TEM (Transmission Electronic Microscope) picture of a PCM device showing a GST film connected to a narrow bottom contact, called heater, and a top thin-film contact [31]. The heater is contacted to one or more select transistors by a metal plug, usually made of tungsten. The select transistor can be a field-effect transistor (FET) as in Fig 6 [31], a bipolar junction transistor (BJT) [10], or a diode-like 2terminal select device [32-36], depending on the required device density in the memory array. In fact, FETs tend to have a larger area due to their planar geometry, thus allow a relatively small device density. On the other hand, 2-terminal selectors, such as polycrystalline silicon p-n junctions [32], ovonic threshold switch (OTS) elements [33] or mixed ionic-electronic conduction (MIEC) devices [34] provide the smallest device area and allow for achieving crosspoint arrays with maximum memory density. A detailed review of PCM cells reported so far in the literature is given in chapter 9.

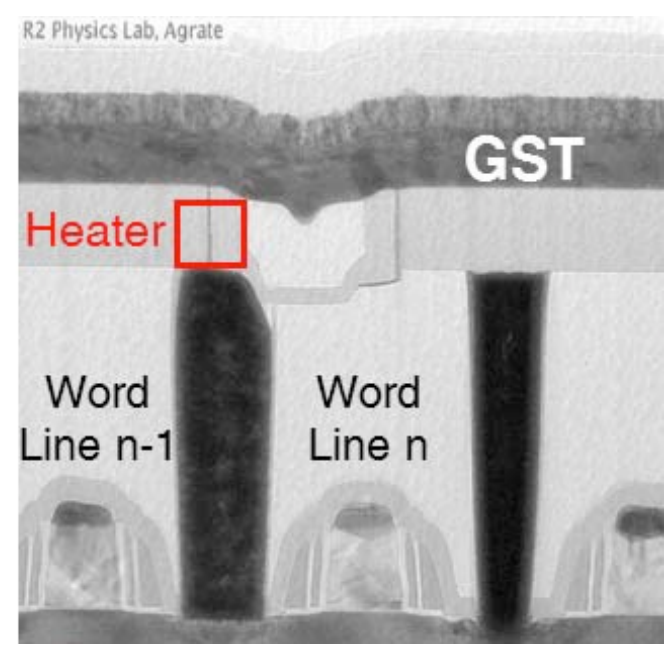

Fig. 6 TEM picture of a PCM device with confined bottom electrode, or heater, and non-confined thin-film GST and top electrode. Each PCM device is connected to 2 transistor acting as select elements for electrical access within the memory array [31].

During the application of a programming pulse, the current flowing in the confined bottom electrode causes Joule heating within the GST volume adjacent to the bottom electrode. For sufficiently intense Joule heating, the GST temperature can exceed the melting point, thus causing local transition to the liquid phase. If the 
decay time of the applied pulse is short enough, typically below $30 \mathrm{~ns}$ [7], the liquid phase is quenched into an amorphous phase, thus inducing reset transition of the PCM. Note that the amorphous volume generally reflects the liquid volume during the reset pulse, thus increases with the amplitude of the voltage pulse [3538]. This enables multilevel programming of the PCM, where different resistance levels correspond to different thickness of the amorphous volume in the GST layer. On the other hand, the set state corresponds to a wholly crystalline phase, typically consisting of polycrystalline fcc structure.

\section{I-V curves for set and reset states}

Fig. 7 shows the measured current-voltage (I-V) curves for a PCM device with confined bottom electrode $[10,39]$. In the measured device, the GST film was deposited by RF sputtering [40]. Top and bottom contacts were consisting of sputtered TiN, where a Ti adhesion layer was inserted between the GST film and the top electrode to minimize the contact interface resistance. The width of the BE was confined to $50 \mathrm{~nm}$ via conventional lithography, whereas the thickness was $5 \mathrm{~nm}$ in the orthogonal direction, thus resulting in a bottom contact area of the PCM device of about $250 \mathrm{~nm}^{2}$. The GST layer had a thickness of $35 \mathrm{~nm}$. Set and reset states in Fig. 7 indicate strongly different conduction behaviors: the current for the set state shows a linear (ohmic) increase with voltage, with resistance $\mathrm{R}=\mathrm{V} / \mathrm{I}$ of about $10 \mathrm{k} \Omega$. On the other hand, the reset state shows a linear increase of current for very low voltage (less than about $0.2 \mathrm{~V}$ ), followed by an almost exponential increase of current for relatively large voltage. A sudden voltage drop, or snapback, occurs at the so-called threshold voltage $\mathrm{V}_{\mathrm{T}}$ of about $1.2 \mathrm{~V}$ in the figure, corresponding to a threshold current $\mathrm{I}_{\mathrm{T}} \approx 3 \mu \mathrm{A}$ for this technology. The voltage snapback is the result of threshold switching, that is a characteristic negative differential resistance (NDR) effect which is generally observed in amorphous phase change materials and OTS $[19,41-50]$. After threshold switching, the I-V curve of the reset state overlaps to that of the set state, most likely due to the crystallization taking place at large current and relatively long times during the DC measurements. Note that at low voltage in the ohmic regime, the reset state displays a resistance of about $10 \mathrm{M} \Omega$, thus resulting in a resistance ratio of about $10^{3}$ between the 2 logic states in the PCM.

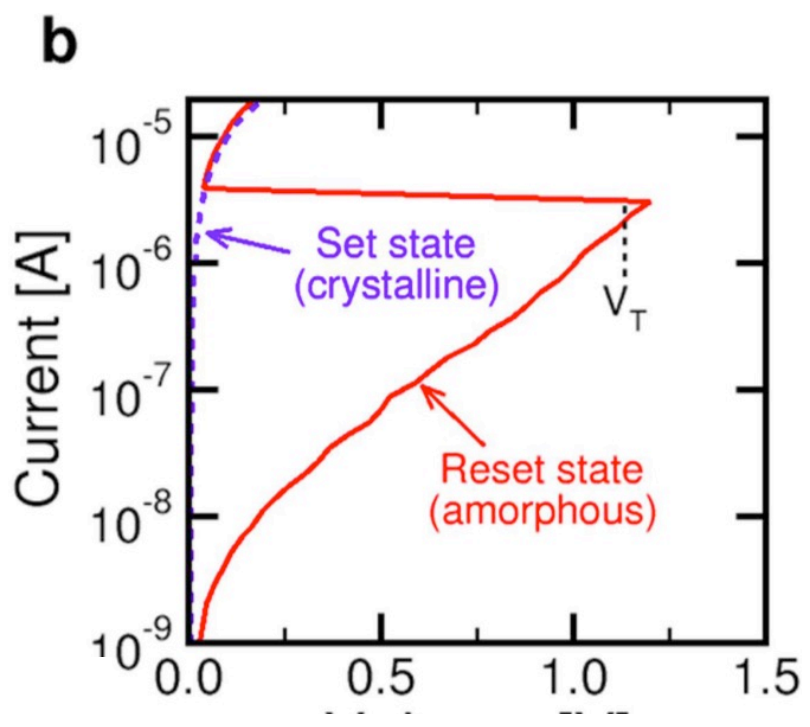

Fig. 7 Measured I-V curves for a PCM device in the reset state (amorphous phase) and set state (crystalline phase) [39]. 
The linear I-V curve of the set state can be attributed to the band structure of the fcc GST, where the degenerate Fermi level causes a large concentration of free carriers contributing to drift transport. The positive activation energy of the fcc structure in Fig. 3 might be explained by the polycrystalline structure of the set state [51], where carriers are thermally-activated in the transport over energy barriers associated to grain boundaries [52]. The variable positive activation energy in the crystalline phase was also attributed to disorderinduced localized states in the crystalline phase, according to the Anderson theory of localization [53].

(a)

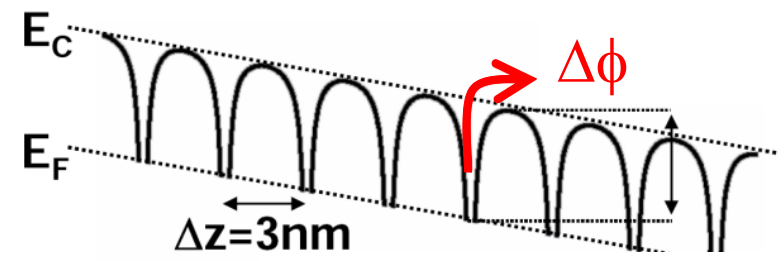

(b)

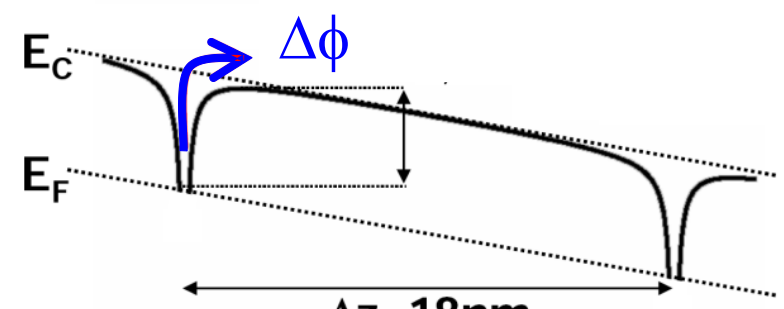

$\Delta \mathrm{z}=18 \mathrm{~nm}$

Fig. 8 Schematic diagram of PF conduction in amorphous GST, comparing the cases where the distance $\Delta z$ between charged localized states is relatively short (a) or relatively long (b) [27].

\section{Poole-Frenkel conduction in the reset state}

The non-linear behavior of the I-V curve of the reset state can instead be attributed to Poole-Frenkel (PF) conduction [27,36,37,47,48]. Fig. 8 schematically illustrates the band diagram under an applied field in the amorphous GST, where carrier transport is due to thermally-excited hopping between positively-charged localized states [54]. In the case of relatively short distance $\Delta z$ between localized states (Fig. 8a), the energy barrier $\Delta \phi(\mathrm{V})$ under an applied voltage $\mathrm{V}$ is given by [47]:

$\Delta \phi(\mathrm{V})=\Delta \phi(0)-\mathrm{qF} \frac{\Delta z}{2}=E_{C}-E_{F 0}-\mathrm{qV} \frac{\Delta z}{2 u_{a}}$

where $\Delta \phi(0)$ is the energy barrier under zero electric field $F, E_{F O}$ is the equilibrium Fermi level, $E_{C}$ is the maximum of the barrier equivalent to the conduction band minimum between 2 localized states, and $u_{a}$ is the thickness of the amorphous volume where all the voltage $V$ is assumed to drop. According to Eq. (2), the energy barrier for PF transport decreases linearly with $\mathrm{V}$, which applies for relatively small $\Delta z$. As a result, the thermally-activated current for relatively large $V$ can be written as [47]:

$\mathrm{I}=\mathrm{qA} N_{T} \frac{\Delta z}{\tau_{0}} e^{-\frac{E_{C}-E_{F 0}}{k T}} e^{\frac{q V \Delta z}{k T 2 u_{a}}}$ 
where $\mathrm{N}_{\mathrm{T}}$ is the concentration of localized states and $\mathrm{T}$ is the local device temperature. Eq. (3) accounts for the exponential increase of I with voltage in Fig. 7. Note that the thermally-activated forward current in the direction of the electric field must be corrected by a reverse current in the opposite direction, which is comparable to the forward contribution at small voltages. After the correction, the current is given by [47]:

$\mathrm{I}=2 \mathrm{qAN} N_{T} \frac{\Delta z}{\tau_{0}} e^{-\frac{E_{C}-E_{F 0}}{k T}} \sinh \left(\frac{q V}{k T} \frac{\Delta z}{2 u_{a}}\right)$

where $N_{T}$ is the density of localized states and $T$ is the local device temperature (I would remove this already explained in the previous equation). Eq. (4) accounts for the linear behavior of current at small voltage, as well as for the exponential current at higher voltage. For relatively long $\Delta z$ (Fig. 8b), the physical picture of conduction remains qualitatively the same, except for the voltage dependence of energy barrier which decreases with the square root of $\mathrm{V}$, thus causing the current to increase exponentially with $\mathrm{V}^{1 / 2}$, namely $\log (\mathrm{I})$ $\sim \mathrm{V}^{1 / 2}$, in line with the traditional PF model of conduction [47]. Both regimes can be experimentally observed in $\mathrm{PCM}$ devices, with the conventional PF $\left(\mathrm{V}^{1 / 2}\right)$ regime arising preferentially for relatively small devices, whereas the $\mathrm{V}$-regime of conduction appearing typically in larger devices [55]. A transition from $\mathrm{V}$ - to $\mathrm{V}^{1 / 2}$-regime can also be seen as a function of time from the reset process, due to the structural relaxation of the amorphous GST, also known as the drift phenomenon [54]. Initially, the I-V curve of the device shows a small- $\Delta z$ behavior with $\log (I) \sim V$, while at long times after reset the conventional PF regime with $\log (I) \sim \mathrm{V}^{1 / 2}$ appears. This can be attributed to the annihilation of localized states caused by the structural relaxation [54].

For a full confirmation of the physical picture of transport in Fig. 8, one should test the model against as many as possible experimental dependencies, such as the dependence on temperature and impact of the programming condition. To this purpose, Fig. 9a shows the measured and calculated I-V curves for T increasing from $25^{\circ} \mathrm{C}$ to $85^{\circ} \mathrm{C}$. There is a good agreement between the calculated I-V curves and the reported data, in both the $\mathrm{V}$ dependence of the individual I-V curves, and the impact of temperature. As $\mathrm{T}$ increases, the current increases quite significantly due to the Arrhenius-type thermal activation in Eq. (4). These data allow to extract the activation energy at variable $V$, from the slope of $\log (I)$ in the Arrhenius plot [47]. Fig. 9b shows the activation energy $E_{A}=d \log (I) / d(1 / k T)$ as a function of $V$, extracted from data and calculations in Fig. 9a. Note that the activation energy can be viewed as the effective value of the energy barrier for carrier transport in the PF process. The decrease of $E_{A}$ at increasing $V$ is thus a clear evidence and confirmation of the PF conduction mechanism in the amorphous phase change material.
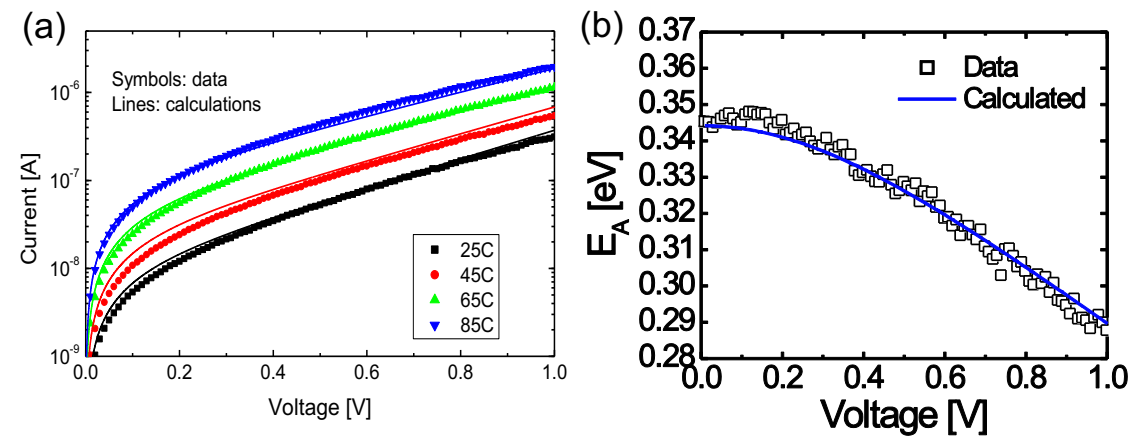

Fig. 9 Measured and calculated I-V curves in the reset state at variable temperature (a) and corresponding activation energy $E_{A}=d \log (I) / d(1 / k T)$ as a function of $V(b)$ [47]. 
(a)

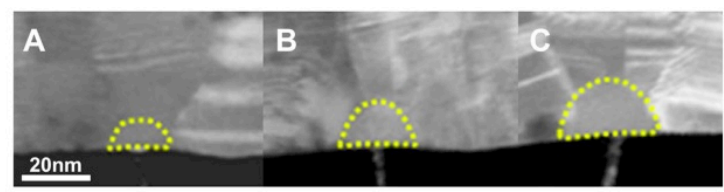

(b)

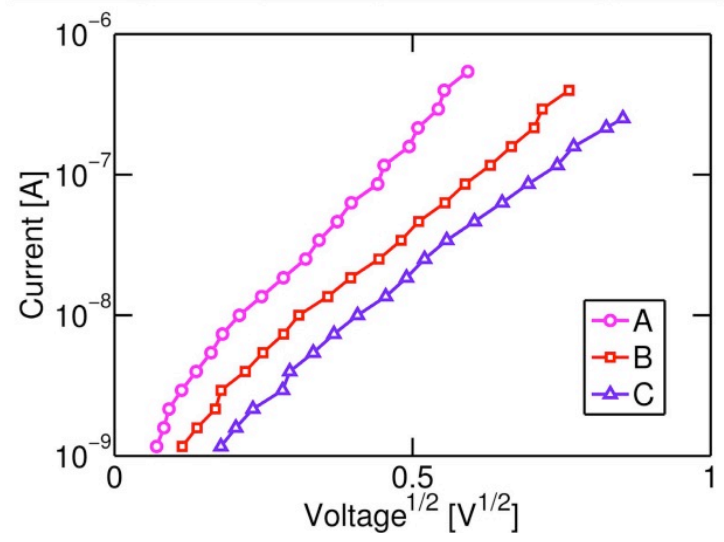

Fig. 10 TEM of the amorphous cap for increasing programming voltage (a) and corresponding measured current as a function of $\mathrm{V}^{1 / 2}(\mathrm{~b})[36]$.

Another compelling evidence of the PF transport mechanism in the amorphous phase is obtained by testing the dependence of the subthreshold slope (STS), given by STS $=d \log (I) / d V$ on the amorphous slayer thickness $u_{a}$. Fig. 10a shows the TEM pictures of the active cell region evidencing the amorphous cap in 3 cells programmed at increasing voltage, while Fig. 10b shows the corresponding I-V curves in the PF plot, where $\log (\mathrm{I})$ is reported as a function of $\mathrm{V}^{1 / 2}$. By calculating the derivative of $\log (\mathrm{I})$ with respect to $\mathrm{V}$ in the long $\Delta \mathrm{z}$ regime, one obtains [36]:

$\mathrm{STS}=\frac{q}{k T} \sqrt{\frac{\mathrm{q}}{\pi \epsilon u_{a}}}$

where $\varepsilon$ is the dielectric constant. Data in Fig. 10b indicate that the STS in the PF plot decreases with increasing $\mathrm{u}_{\mathrm{a}}$, in qualitative agreement with Eq. (5), thus supporting the PF model of conduction. Further support to the PF model was provided by demonstrating the inverse dependence of STS on T according to Eq. (5) [56]. 


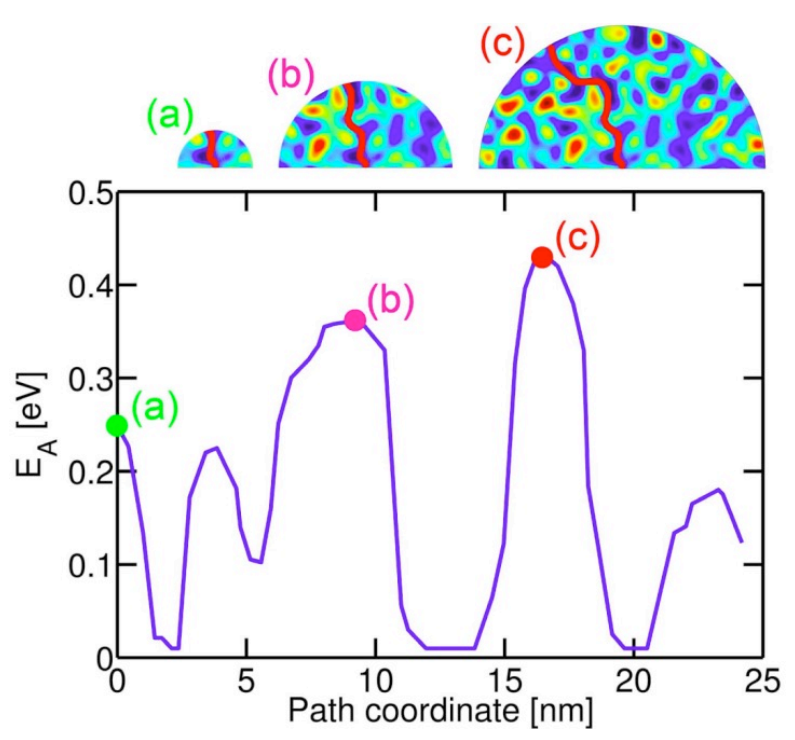

Fig. 11 TEM of the amorphous cap for increasing programming voltage (a) and corresponding measured current as a function of $\mathrm{V}^{1 / 2}(\mathrm{~b})[36]$.

Further analysis of the I-V curves at variable thickness allows to evidence energy landscape effects, due to the variation of energy barrier resulting from the disordered structure $[36,57,58]$. In particular, the energy landscape is at the origin of the thickness dependent resistivity, where amorphous GST resistivity increases with thickness due to the percolative nature of PF conduction in the energy landscape. An important implication of the energy landscape effect is the non-linear increase of resistance with thickness as the thickness of the amorphous cap increases in the PCM device [57]. This is at the basis, for instance, of the gradual increase of resistance along the programming curve, where an increase of the programming voltage leads to a gradual increase of resistance corresponding to the amorphous volume formed after quenching from the melt. The origin of this thickness-dependence resistivity is explained by Fig. 11, showing the calculated potential energy along the electron percolative path. The potential energy can be viewed as the energy barrier $E_{A}$ which the electrons must overcome by thermal excitation in the PF transport. For a thin amorphous cap (a), the percolative path is short, thus $E_{A}$ will statistically display relatively small values. As the amorphous cap is increased as in (b) or (c), there are increasing chances to cross a high $E_{A}$ point in the percolative path. As a result, $E_{A}$ (hence the measured resistivity) increases with the amorphous cap thickness. Energy landscape phenomena should thus be taken into account for projecting the thickness scaling of PCM.

\section{Threshold switching}

The most distinguishing aspect of the conduction characteristics in the amorphous chalcogenide is the threshold switching, that is the abrupt change of conductivity occurring at the threshold voltage $\mathrm{V}_{\mathrm{T}}$ in Fig. 7 [4150]. Threshold switching generally arises due to bi-stable S-type NDR, where 2 different currents can be achieved for a given voltage within a restricted voltage range. This is schematically illustrated in Fig. 12a showing the typical I-V curve with an OFF state at low current and an ON state at high current in correspondence of the same voltage. The bi-stable behavior can be understood by the fact that the same 
voltage drop across the same semiconductor thickness can be sustained with 2 different band diagrams: in the OFF state (Fig. 12b), the voltage drops homogeneously across the device thickness, resulting in a relatively low electric field inducing equilibrium carrier transport, such as the PF conduction process in Fig. 8. On the other hand, the ON state (Fig. 12C) consists of a non-equilibrium configuration of bands with heavily nonhomogeneous distribution of the electric field. The field is extremely high within an initial dead layer, where the high field induces injection of carriers in the following ON region, where the field is instead relatively low thanks to the large concentration of injected carriers. Alternatively, injection might lead to transfer of carriers to higher energy, thus enhancing their mobility. Current continuity in the ON and dead layers is ensured since the current is proportional to the product of carrier density, carrier mobility and electric field, thus a high field and low concentration/mobility in the dead layer is balanced by the low field and high concentration/mobility in the ON layer.

(a)

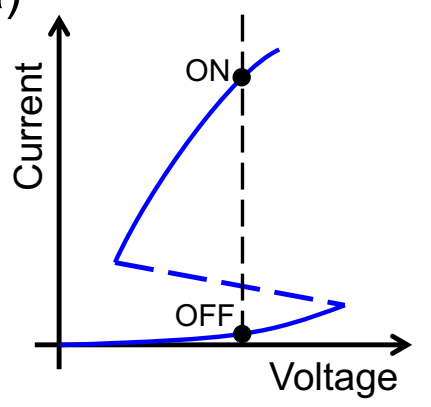

(b) OFF state

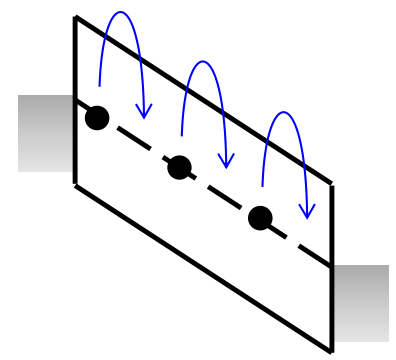

(c) ON state

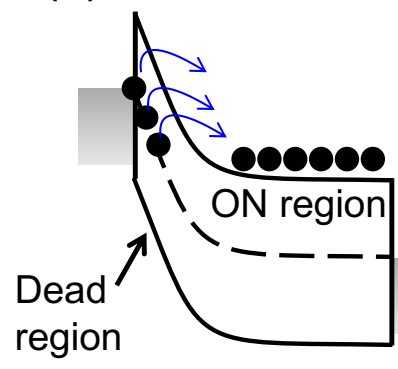

Fig. 12 Schematic illustration of NDR mechanism at the basis of threshold switching, showing the typical I-V characteristics for NDR (a) and the band diagrams for the OFF state (b) and the ON state (c).

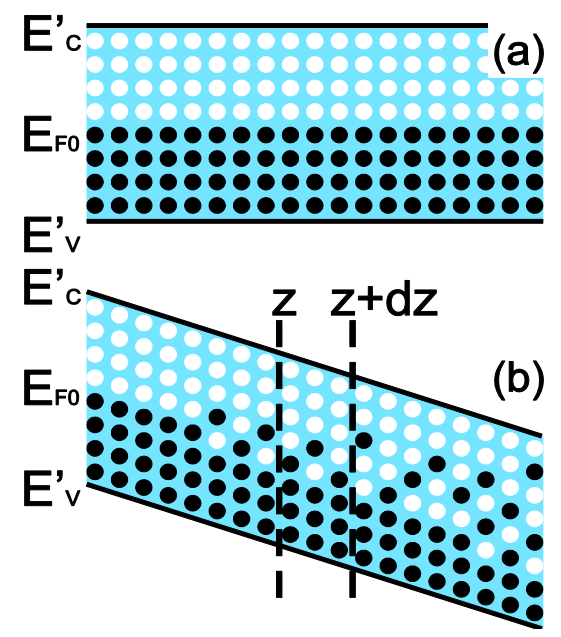

Fig. 13 Schematic band diagram illustrating the mechanism of energy gain describing threshold switching. at zero voltage, electrons occupy all states below the equilibrium Fermi level $\mathrm{E}_{\mathrm{F} 0}(\mathrm{a})$, while the application of a relatively high voltage cause the gain of energy of carriers due to (b) [48]. 
The injection mechanisms in the dead layer of Fig. 12c can arise from different mechanisms, such as tunneling [47] or even PF conduction itself [48], as a result of the carrier heating phenomenon illustrated in Fig. 13. In fact, the PF mechanism involves the thermal excitation of carriers over an energy barrier for transport. At low field (Fig. 13a), carriers can relax their energy after a hopping event, thus the energy distribution of carriers remains that of the equilibrium state, where roughly all states are filled below $E_{F}$, while roughly all states are empty above $E_{F}$. One should recall that a large concentration of localized states exists in the amorphous semiconductor band gap, and sustains the PF transport process. At sufficiently-high electric fields (Fig. 13b), there is no sufficient time for the carrier energy to relax between the capture and emission events [59-61], thus carriers can gain a significant amount of energy resulting in a hot (non-equilibrium) electron distribution. This is similar to the hot electron effects arising in a FET channel at high electric fields [62], except that the nonequilibrium distribution mostly affects the density of carriers in localized states, rather than the in the band of extended states. Due to the exponential energy dependence of electron mobility in the PF transport, the conductivity can be significantly enhanced, thus resulting in a reduced voltage drop in the region where carriers have high energy, in agreement with the simplified conceptual picture of Fig. 12c. Note that this picture only assumes PF at low/high field as the origin of threshold switching, thus is capable of explaining threshold switching in a wide class of materials, e.g., phase change chalcogenides, OTS materials, and in principle any other material sharing the PF mechanism as the dominant conduction process [48].

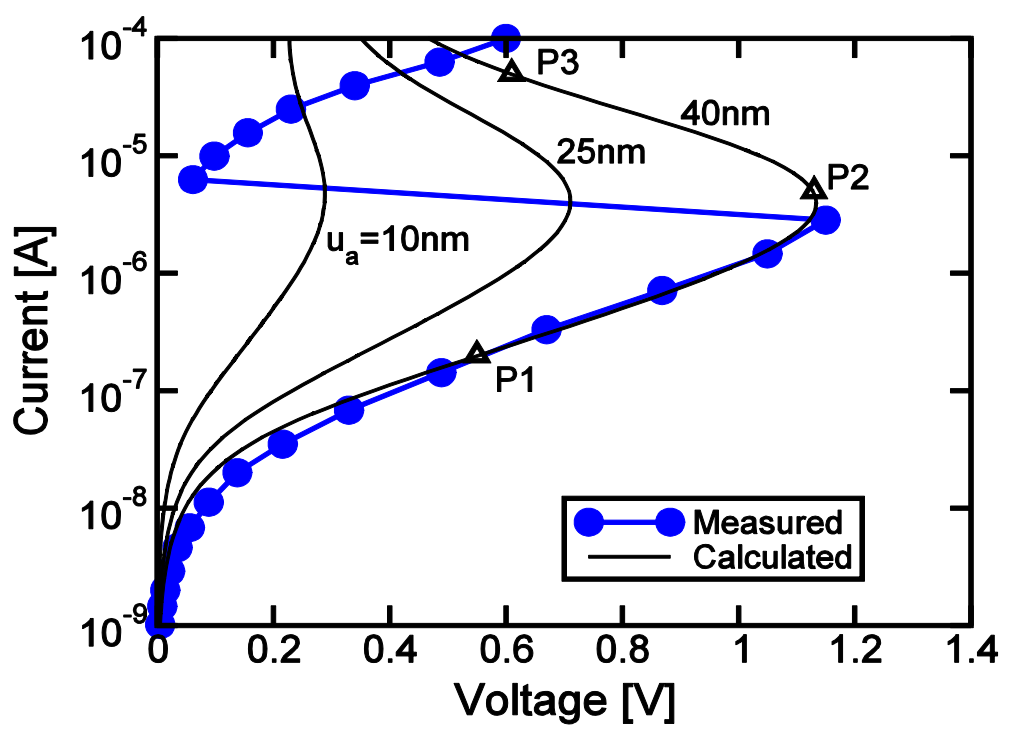

Fig. 14 Measured and calculated I-V curves for a PCM device, showing threshold switching induced by S-type NDR. Calculations were performed for variable thickness of the amorphous layer [48].

Based on the physical picture in Fig. 13, a numerical model was developed to mathematically reproduce the NDR and consequent threshold switching [48]. According to Fig. 13, the model relies on an energy balance for carriers (electrons in the figure) in a layer of infinitesimal thickness $d z$. The energy flow input into the infinitesimal layer can be written as $J(z) E_{F}(z) / q$, namely the carrier flow multiplied by the average carrier flow 
equal to the local quasi Fermi energy $E_{F}$. The latter can be different from the equilibrium value $E_{F 0}$ as a result of the energy gain induced by the field. Within the layer, carriers receive a contribution to the energy equal to the electrostatic energy loss, namely $\mathrm{dE}_{\text {gain }}=\mathrm{qFdz}$. On the other hand, scattering with phonons and defects leads to an energy loss given by $d E_{\text {loss }}=\left(E_{F}-E_{F 0}\right) n_{T} d z / \tau_{\text {rel, }}$, where $n_{T}$ is the effective density of trapped carrier contributing to the energy flow and $\tau_{\text {rel }}$ is a characteristic relaxation time constant describing the carrier tendency to thermalize their distribution from non-equilibrium [48]. By combining the 4 contributions (energy input, energy output, energy increase and energy loss in the layer), one obtains a differential equation for the carrier energy, given by:

$\frac{d E_{F}^{\prime}}{d z}=\mathrm{qF}-\frac{\mathrm{q} n_{T}}{J \tau_{0}} \frac{E_{F}^{\prime}-E_{F}}{\tau_{r e l}}$

which allows to calculate the $E_{F}$ profile for any applied current density J [48]. Eq. (6) can be solved by explicating the relationship between local field $F$ and current density, e.g., based on the PF transport equation, under the continuity condition that $\mathrm{J}$ remains uniform over the entire thickness $\mathrm{u}_{\mathrm{a}}$.

Fig. 14 shows the I-V curves obtained from measurements and calculations using Eq. (6) for various values of the amorphous GST thickness $u_{a}$. Calculations agree with the measured data for a thickness $u_{a}=40 \mathrm{~nm}$, which agrees with the typical thickness of the amorphous cap in a PCM device after full reset operation. Calculations show the characteristic S-type NDR behavior as a result of the increase of the carrier energy $\mathrm{E}_{\mathrm{F}}$ as described in Fig. 13. Three points are marked in Fig. 14, correspond to subthreshold condition (P1), where the field is too low to induce any significant energy increase of carriers, thus $\mathrm{E}_{\mathrm{F}}=\mathrm{E}_{\mathrm{FO}}$ over the whole thickness. At the threshold point (P2), the energy gain is sufficiently high to induce a significant boost of conductivity in the ON region. Starting from this condition, any further increase of current density results in an increased field in the dead layer, a higher energy gain in the ON region, and a correspondingly smaller field in the ON region. As a result, the voltage decreases at increasing current, which is at the basis of the NDR behavior in Fig. 14. Point P3 at high current in the NDR regime is an example of this situation, which can be visualized by Fig. 12c while P1 at almost equal voltage drop can be represented by Fig. 12b. The threshold point P2 thus marks the conditions (current density and voltage $\mathrm{V}_{\mathrm{T}}$ ) for which the energy gain of the electron population reaches a critical value, around kT, which enable sufficient enhancement of conductivity to trigger the NDR. A detailed analysis shows that a fixed energy gain is equivalent to a constant power density $P^{\prime \prime \prime}=$ FJ within the device. This condition can be used for a fast prediction of threshold point in PCM devices.

Note again the large generality of this description which results from the energy balance of Eq. (6) without invoking any additional mechanisms except PF transport under non-equilibrium distribution of energy induced by the high electric field. Note also that the contribution of additional mechanisms, such as Joule heating [38], impact ionization $[19,45]$, polaron instability [46], etc., may not be ruled out from this analysis. A different view is proposed in chapter 4 where carrier generation-recombination balance is at the basis of the switching event, which can be conveniently implemented in a finite element based device simulator tool.

Fig. 15a shows the calculated I-V curves for variable temperature (a). A reference experimental characteristic measured at $300 \mathrm{~K}$ for GST is also shown for reference. The NDR region of the I-V curves was deleted for clarity and the I-V curves were shown only up to the threshold voltage $V_{T}$. Calculations show that the threshold voltage $V_{T}$ decreases with increasing $T$, as a result of the Arrhenius-type increase of subthreshold PF leakage. In 
fact, to maintain a constant power density for switching, $V_{T}$ decrease as the subthreshold current increases. This results in an approximately linear decrease of $V_{T}$ with $T$.

Fig. $15 \mathrm{~b}$ shows the calculated I-V curves for variable $\mathrm{E}_{\mathrm{C}}-\mathrm{E}_{\mathrm{FO}}$, which corresponds to about half of the band gap assuming that the Fermi level is located at mid gap. The PF current decreases at increasing $\mathrm{E}_{\mathrm{C}}-\mathrm{E}_{\mathrm{F} 0}$, thus causing $V_{T}$ to increase as a result of the constant-power rule. This is generally demonstrated experimentally, as phase change materials with a low leakage current display a relatively large activation energy for conduction, as well as a relatively large $\mathrm{V}_{\mathrm{T}}$. This provides relevant design guidelines to engineer the phase change material to match a certain circuit requirement for $\mathrm{V}_{\mathrm{T}}$.

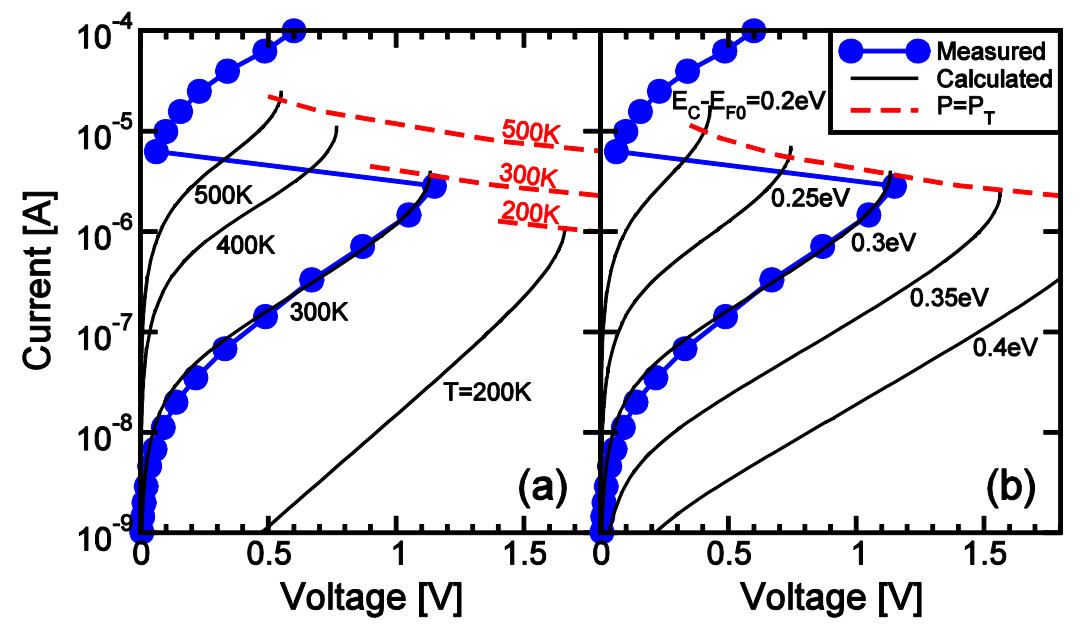

Fig. 15 Measured and calculated I-V curves for a PCM device for variable temperature (a) and variable $\mathrm{E}_{\mathrm{C}}-\mathrm{E}_{\mathrm{F} 0}$ (b) [48]. Lines at constant power density are also shown for reference of the constant-power rule of threshold switching.

\section{Switching dynamics}

While most modeling and characterization studies aim at describing the DC behavior of switching, namely the threshold voltage and current under quasi-static DC-type measurement conditions, time-dependent analysis of switching has been attempted less frequently. The time-dependent switching dynamics can be studied with reference to 2 fundamental parameters: the delay time $\tau_{D}$, namely the time needed to initiate the switching process since the first application of the voltage, and the switching time, $\tau_{s}$, namely the time for the current to rise from the subthreshold level to the ON state.

The switching time can be viewed as the time to switch from the OFF-state (uniform field) configuration in Fig. $12 \mathrm{~b}$ to the ON-state configuration (non-uniform field) in Fig. 12c, requiring electron heating and establishment of the high-energy/high-concentration conditions in the ON region. This evolution is governed by a positive feedback effect, in that a momentary increase of carrier energy results in a higher conductivity in the ON-region, thus causing a higher current and a corresponding increase of the voltage drop across the dead region to sustain the increased current. A higher electric field across the dead region results in (i) an increase of 
the energy in the OFF region, causing a further increase of current, and (ii) a decrease of the voltage drop across the ON region to maintain the constant voltage across the PCM device. Ideally, such a positive feedback loop would lead to an indefinite increase of current causing final destructive failure of the device, but in practice the series resistance in the PCM device, e.g., caused by the confined bottom electrode, would limit the maximum current, thus allowing to reach a final steady state as sketched in Fig. 12c.

Attempts to measure the switching time were not successful to date, possibly due to the positive-feedback loop and the correspondingly very fast switching transient. Calculations of the switching time were carried out by using an analytical model of threshold switching based on tunneling from gap states to the conduction band [47]. Fig. 16 shows the calculated I-V curves with the transient trajectory during time-dependent switching (a), and the calculated current (top), electric field (center) and carrier concentration (bottom) as a function of time [63]. The I-V trajectory during switching assumes a load resistance $R_{L}=3 k \Omega$ in series, and includes 3 representative points, corresponding to initial equilibrium conditions in the OFF state (i), transient state during the switching event (ii) and final ON state at high currnet (iii). At time zero, the device is biased with an applied voltage in the OFF state (i) with subthreshold current flowing and a negligible concentration of excess carriers $\delta \mathrm{n}_{\mathrm{T} 2}$ in the $\mathrm{ON}$ region. Equal electric fields are present in the dead and $\mathrm{ON}$ regions due to the equilibrium conditions. However, the OFF-state field injects a gradually increasing concentration of excess carriers in the ON region as apparent from the transient of $\delta n_{T 2}$ in Fig. $16 \mathrm{~b}$ (bottom). As $\delta n_{T 2}$ becomes comparable to the preexisting (equilibrium) carrier concentration, the current start to increase, resulting in the switching event. As the current increases during switching (ii), the electric field in the dead region Foff increases, while the electric field in the ON region $F_{O N}$ decreases, thus establishing the non-uniform field distribution in Fig. 12c. Steady state is eventually reached in the ON state (iii).

(a)

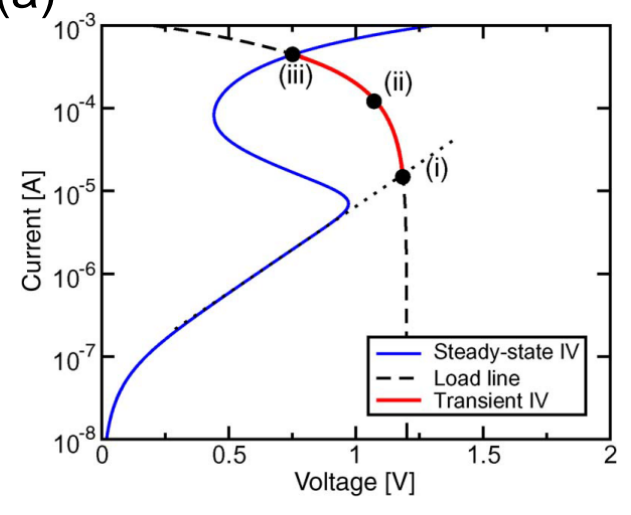

(b)

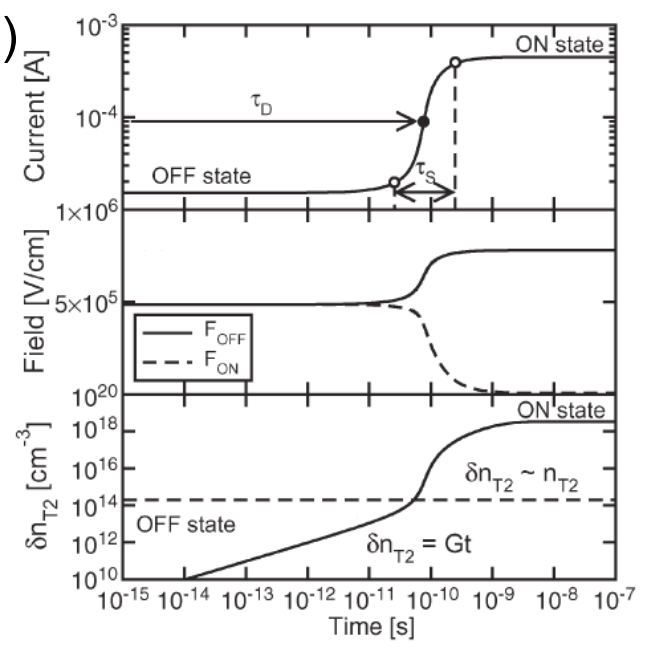

Fig. 16 Calculated I-V curves and trajectory during the switching transient (a) and corresponding current (top), electric field (center) and carrier concentration (bottom) as a function of time (b) [63]. Note the gradual increase of excess carriers $\delta \mathrm{n}_{\mathrm{T} 2}$ which triggers the switching event with the corresponding steep rise of current, $\delta \mathrm{n}_{\mathrm{T} 2}$ and discrimination of electric fields in the dead and ON regions. 
The simulated current transient in Fig. 16b (top) allows to discriminate between delay and switching transients: the delay time $\tau_{D}$ can be defined as the time for current to reach half of the switching swing, while the switching time $\tau_{\mathrm{s}}$ can be defined as the net time for the current to switch from the OFF to the ON state, represented by $10 \%$ and $90 \%$ of the whole current swing. Physically, the delay transient in the simulation is described by the time to build up a concentration of extra carriers $\delta n_{\mathrm{T} 2}$ comparable to the equilibrium concentration, while the switching time is the time for the positive-feedback driven steep increase of the current. Note that $\tau_{\mathrm{D}}$ and $\tau_{\mathrm{S}}$ are both extremely short, thus posing fundamental difficulties in time-resolved measurements of these times.

More detailed analysis indicates a key role of the parasitic capacitance which is inevitably present in any PCM device during measurements [63]. In general, a parasitic capacitance $\mathrm{C}$ of few pF may be expected to affect a conventional experimental setup for electrical measurements [64]. The parasitic capacitance affects the measurements in 2 ways: (i) the delay time is increased by the RC delay in the circuit, resulting in longer $\tau_{D}$ given approximately by the time to build-up a charge $Q_{i}=C V_{A}$ within the capacitance, and (ii) the current increases in excess of what expected from the load-line trajectory in Fig. 16, due to the parasitic overshoot $[63,64]$. The latter phenomenon is due to the fact that the time to discharge the parasitic capacitance $C$ is approximately given by $R_{L} C$, namely, the $R C$ time of the circuit. Since this is generally much longer than $\tau_{S}$, the voltage across the PCM remains constant during switching, instead of adjusting to the lower value according to the load line in Fig. 16a. As a result of the larger voltage across the PCM, switching time is shorter than the calculated $\tau_{\mathrm{s}}$ in Fig. 16b, which assumed a decreasing voltage during switching. also, the maximum current reached after switching is larger than the case of zero capacitance, which accounts for the term 'overshoot'.
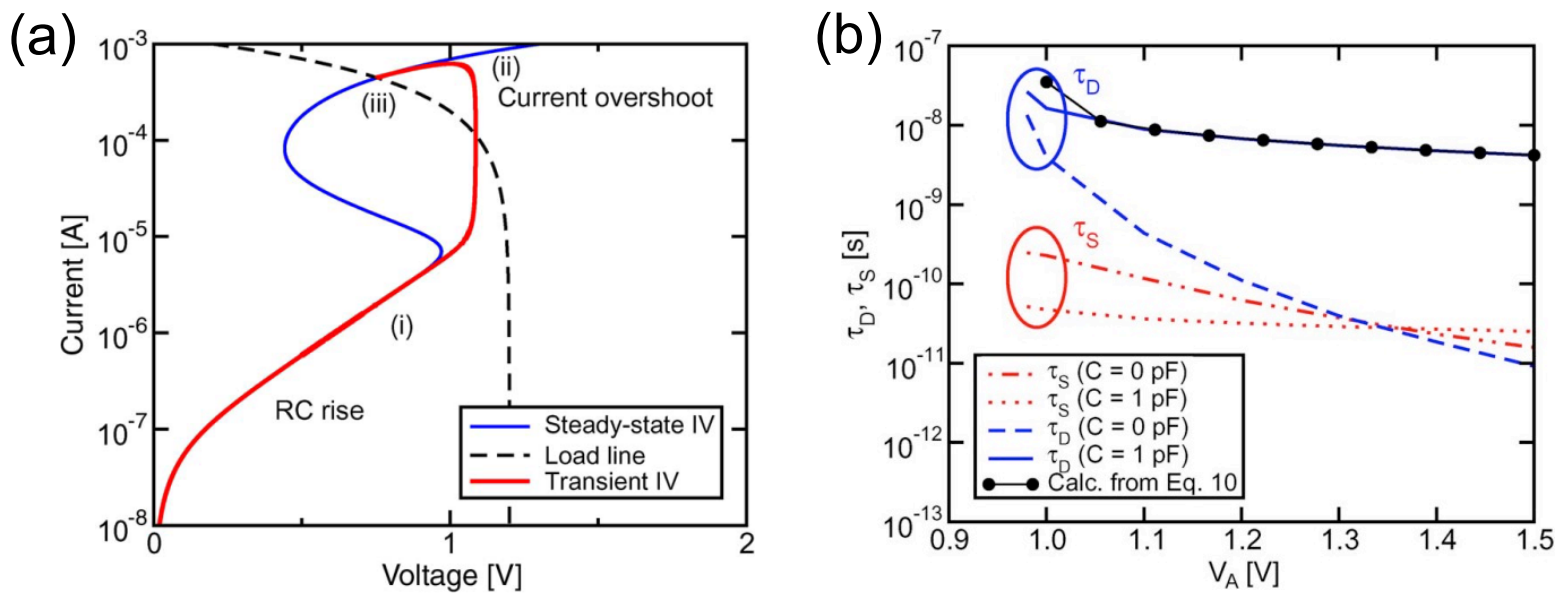

Fig. 17 Calculated I-V curves and trajectory during the switching transient for non-zero parasitic capacitance (a) and calculated $\tau_{\mathrm{D}}$ and $\tau_{\mathrm{S}}$ as a function of applied voltage (b) comparing situations with and without parasitic capacitance [63].

Fig. 17 shows the calculated I-V curve and the switching trajectory for a PCM device with a parasitic capacitance $\mathrm{C}=1 \mathrm{pF}(\mathrm{a})$ and the corresponding $\tau_{\mathrm{D}}$ and $\tau_{\mathrm{S}}$ as a function of applied voltage with and without a 
parasitic capacitance $\mathrm{C}=1 \mathrm{pF}(\mathrm{b})$. The switching characteristics shows a steep transition with constant voltage taking place in a time $\tau_{s}$, followed by a readjustment of voltage taking place in a longer time $R_{L} C$. Due to the first stage of vertical switching, the current shows an overshoot effect. The parasitic capacitance results in a longer delay time which is dominated by the RC delay according to the formula:

$\tau_{D}=R_{L} \operatorname{Clog} \frac{1}{1-V_{T} / V_{A}}$

which is calculated as the time to reach close to $V_{T}$ in a $R_{L} C$ circuit under an applied voltage $V_{A}$ [63]. Calculation from Eq. (7) are also shown in the figure for comparison. The switching time instead is decreased by the parasitic capacitance due to the constant voltage during the switching transient. Note however that the switching time is always in the sub-nanosecond range, which makes it difficult to attempt an experimental evaluation of the switching time.

More detailed studies of the delay time indicate that there are 2 regimes, namely RC-limited delay time at relatively high voltage, as shown in Fig. 17 and described by Eq. (7), and the fluctuation-limited delay time at relatively low voltage [65]. Fig. 18a shows the measured voltage across the PCM during switching at relatively low voltage close to $\mathrm{V}_{\mathrm{T}}[66]$. The sharp decrease of voltage in the figure marks threshold switching, as the PCM resistance decreases thus changing the voltage drop in the voltage divider circuit shown in the inset. In these type of measurements, threshold switching occurs at relatively long times, i.e., in the few $\mu$ sime range, compared to the few ns range in Fig. 17, where the shorter delay was due to the higher voltage overdrive across the device. Three repeated measurements are shown in Fig. 18a, indicating a broad distribution of $\tau_{D}$ due to stochastic switching. A detailed analysis of the distribution of $\tau_{D}$ at variable voltage indicates a Weibull distribution with relatively low shape factor, i.e., the slope of the distribution on the Weibull plot [65]. This behavior can be understood by current fluctuations obeying $1 / \mathrm{f}$ power spectral density (PSD), where a 'lucky' fluctuation above a certain threshold current $I_{T}$ triggers threshold switching. Simulations by $1 / f$ current noise models support this picture in terms of both the statistical distribution and the average value of $\tau_{D}$ as a function of the applied voltage $V_{A}$, as shown in Fig. 18b [66]. Data and calculations indicate an extremely steep slope of about $13 \mathrm{mV} / \mathrm{dec}$. This type of analysis allows for a non-ambiguous definition of the threshold voltage $V_{T}$, as the voltage marking the separation the fluctuation-limited regime of $\tau_{D}$ and the high-voltage regime which is controlled by either RC delay, or by the build-up of the non-equilibrium distribution of carriers in the PCM material. The accurate understanding of delay and switching phenomena in Fig. 18 allows for a careful design of PCM in several conditions (read, write, disturb) and applications, such as memory [8-10], logic [67-69] and non-Boolean computation for future data-intensive computing memories [70-72]. 

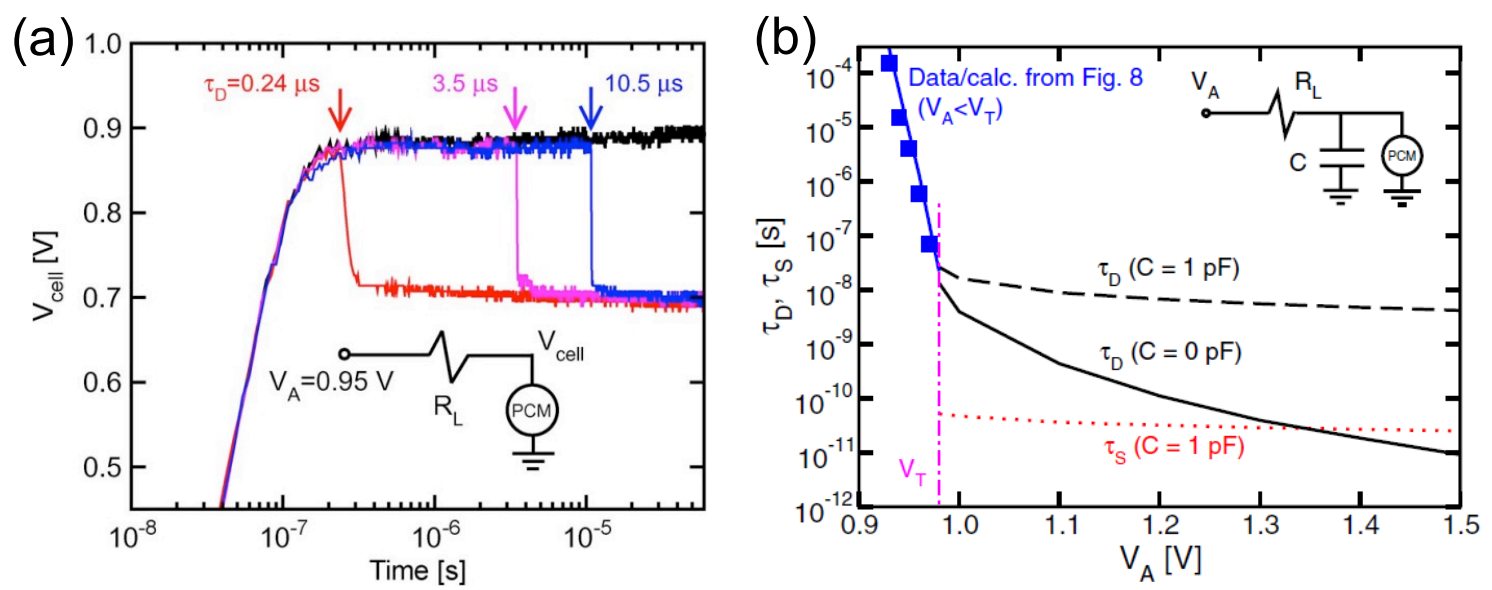

Fig. 18 Measured voltage $\mathrm{V}_{\text {cell }}$ across the PCM device (see inset) as a function of time indicating stochastic switching with variable delay time $\tau_{\mathrm{D}}$ (a) and average $\tau_{\mathrm{D}}$ as a function of applied voltage $\mathrm{V}_{\mathrm{A}}$, compared with calculated $\tau_{\mathrm{D}}$ and $\tau_{\mathrm{S}}$ at various parasitic capacitance $\mathrm{C}(\mathrm{b})[66]$.

\section{Time-dependent transport}

While threshold switching describes the time-dependent transition from high resistance to low resistance due to the bistable nature of the conduction characteristic, resistance drift is a physical process responsible for the transition to high resistance. This is shown in Fig. 19a reporting the resistance for a PCM in the amorphous phase as a function of time measured with respect to the reset pulse [27]. The resistance increases according to a power law, namely [73-75]:

$R=R_{0}\left(\frac{\mathrm{t}}{t_{0}}\right)^{v}$

where $R_{0}$ and $t_{0}$ are constant, and the exponent $v$ is about 0.1 . The set state resistance is also shown in the figure, indicating a stable resistance with negligible drift. Resistance drift in the amorphous phase can be explained by structural relaxation taking place in the non-equilibrium disorder phase, as illustrated in Fig. 19b. Here, defects remained from the liquid phase after quenching represent high-energy configurations, which the system tends to anneal by structural relaxation. For instance, a dangling bond relaxes by fixing itself to an atom to complete a chain. Defects which might play a critical role in the structural relaxation of amorphous GST are wrong bonds, such as Ge-Ge bonds and chains which are largely unstable [76]. Structural relaxation is universally observed in disordered materials, such as amorphous Si [77, 78], amorphous Ge [79], amorphous $\mathrm{SiC}$ [80], and amorphous hydrogenated carbon [81]. Relaxation should not be viewed as a phase transition, since the structure remains amorphous and disordered, while the resistance and band gap increase [74,75]. In fact, in contrast to crystallization, the energy barrier $E_{A}$ for structural relaxation in Fig. 19b belongs to a wide distribution, from relatively small energies to large energies close to the crystallization energy barrier, e.g., about $2.6 \mathrm{eV}$ for GST. As a result, structural relaxation is seen even at room temperature, as shown by the resistance drift in Fig. 19a, due to the relatively small energy barriers in the distribution of $E_{A}$. The broad distribution of energy barriers is at the origin of the power law time dependence of resistance drift, based on the Arrhenius relationship between transition time and $E_{A}[82]$. 


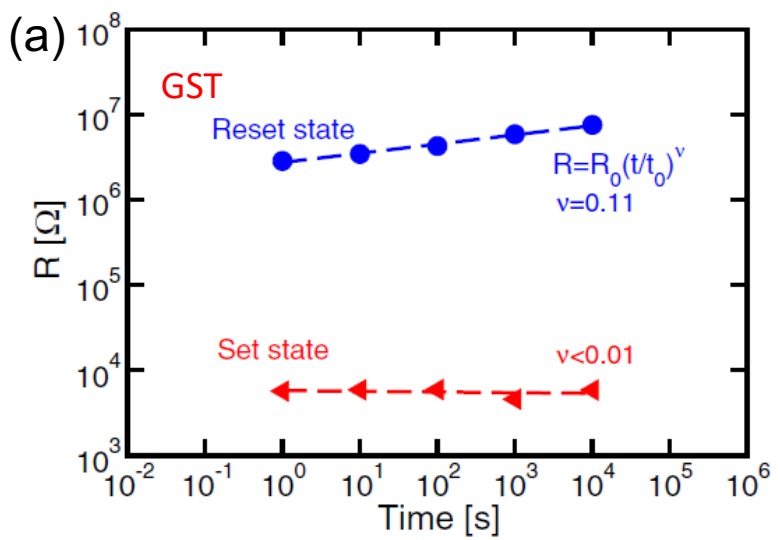

(b)

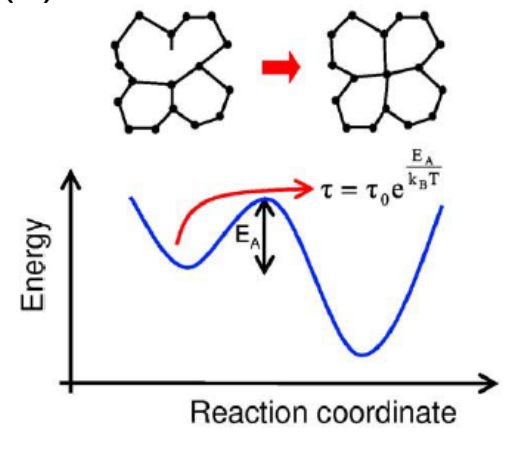

Fig. 19 Measured resistance $\mathrm{R}$ as a function of time after programming (a) and schematic illustration of structural relaxation (b). The power law drift of resistance for the reset state is due to structural relaxation, where defects are annealed by thermally-accelerated relaxation to lower energy states [73].

Structural relaxation results in a consolidation of bonding in the amorphous state, which is reflected by an increasing band gap [40,54] and a correspondingly decreasing dielectric constant [27]. The increased band gap leads to a higher energy barrier for PF conduction, thus causing an increase of resistance and a decrease of leakage current. The lower leakage current also causes a higher threshold voltage, which shows a drift behavior where $V_{T}$ increases linearly with the logarithm of time $[55,75]$. In general, the increase of the reset state resistance does not raise any reliability issue, as the resistance window between set and reset states increase with time. However, the increases $V_{T}$ may eventually make set-state programming difficult, if $V_{T}$ increases above the nominal voltage for set process. This possible problem is easily fixed by adjusting the programming voltage to overcome possible $V_{T}$ drift effects. The main impact of drift on PCM reliability is seen in the case of multilevel storage, where the PCM is programmed at more than 2 levels to enable the storage of multiple bits per cell. For instance, 4 resistance levels, corresponding to set, reset, and 2 intermediate resistance levels, allow the representation of 2 bits in an individual physical cell. Due to the presence of a mixed phase in the intermediate states, resistance displays a measurable drift [83], thus causing broadening of the programmed distributions with time which makes multilevel storage difficult in PCM. In some PCM materials, such as Ge-rich GST which is designed to achieve a higher crystallization temperature for embedded memory and automotive applications, the set state also displays a significant drift due to the presence of substantial disordered phase after set transition [31,52]. Set-state drift may result in a resistance window closure that may negatively affect reliability.

To overcome the resistance drift problem in PCM, some solutions were proposed at the level of both $\mathrm{read} /$ program algorithms, and cell architecture. Fig. 20 shows the measured $\mathrm{R}$ as a function of time for a PCM in the reset state annealed at room temperature or at $120^{\circ} \mathrm{C}$. The resistance was measured at a given read current $I_{\text {read, }}$ instead of a fixed read voltage, and measurement results are shown for increasing $I_{\text {read. }}$. As $I_{\text {read }}$ increases, the resistance value decreases, as a result of the exponential-like increase of the PF current. Most importantly, resistance drift decreases significantly for increasing $I_{\text {read, }}$ which can be again understood by the 
exponential-like increase of the current. In fact, assuming that the current is given by $I=I_{0} \exp \left(V_{\text {read }} / V_{0}\right)$, and assuming that drift is simply described by a decrease of $I_{0}$, the relative resistance increase between time $t_{1}$ and time $t_{2}$ is given by [54]:

$R\left(t_{2}\right) / R\left(t_{1}\right)=\log \left(I_{\text {read }} / I_{0}\left(t_{2}\right)\right) / \log \left(I_{\text {read }} / I_{0}\left(t_{1}\right)\right)$,

which tends to $R\left(t_{2}\right) / R\left(t_{1}\right)=1$ for $I_{\text {read }}>>I_{0}\left(t_{1}\right)>I_{0}\left(t_{2}\right)$, thus resulting in a strong attenuation of the resistance drift with respect to the case of small $I_{\text {read, }}$ of the case of read at a constant voltage, where $R\left(t_{2}\right) / R\left(t_{1}\right)=$ $\mathrm{I}_{0}\left(\mathrm{t}_{1}\right) / \mathrm{I}_{0}\left(\mathrm{t}_{2}\right)[54]$. Similar algorithms based on alternative read metrics were proposed to minimize drift and allow multilevel cell storage in PCM [84].

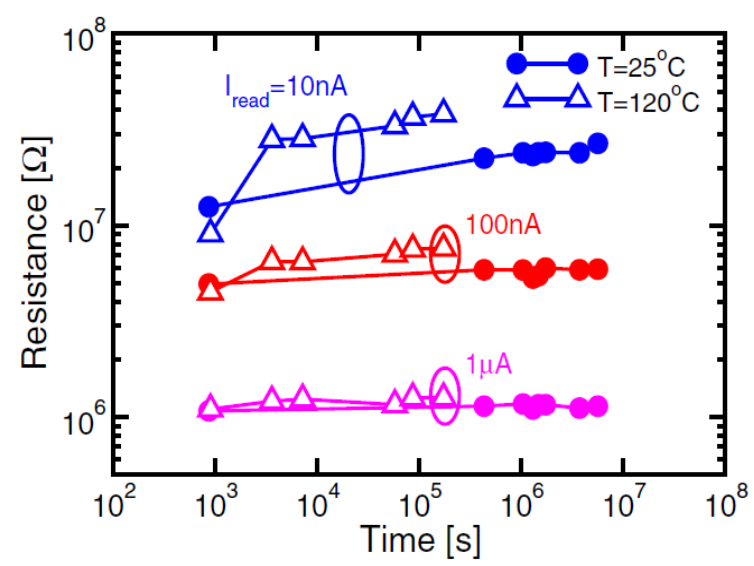

Fig. 20 Measured resistance $\mathrm{R}$ as a function of time after programming (a) and schematic illustration of structural relaxation (b). The power law drift of resistance for the reset state is due to structural relaxation, where defects are annealed by thermally-accelerated relaxation to lower energy states [27].

Drift can also be mitigated by adopting alternative programming mechanisms, such as the bipolar switching. In bipolar switching, different resistance states are obtained by inducing ionic migration, instead of a real phase change [39]. In particular, the high resistance state is obtained by applying a high negative voltage at the top electrode, resulting in a depletion of cation species $(\mathrm{Ge}, \mathrm{Sb})$ at the bottom electrode contact. As the high resistance does not rely on an amorphous region, structural relaxation and drift are fully suppressed in the reset state [39]. Alternative cell architectures have also been proposed to solve the drift problem. For instance, a core-shell structure of the PCM, where a phase change material core pillar is surrounded by a metallic surfactant layer shell, was shown to display less drift, as a result of the parallel current path in the surfactant layer [85]. In fact, the current can sneak around the amorphous region through the surfactant layer, thus preventing any impact of structural relaxation to resistance. The drift coefficient could thus be significantly reduced, although the resistance window also decreases as a result of the surfactant conduction path with relatively low resistance. Combining material, device, and algorithm solutions might enable reliable multilevel storage for ultra-high density PCM in the future. 


\section{Summary}

PCM operation during read and write relies on a careful engineering of the interplay between electrical, thermal, and structural phenomena at the nanoscale. In this interplay, sub-threshold conduction, threshold switching and ON/OFF state transport properties dictate most of the PCM working conditions. This chapter has reviewed the most significant PCM electrical properties affecting resistance window, write and read voltage values, and dynamic response. It is shown that the resistance window relies on the band structure of the phase change semiconductor material in the crystalline and amorphous phase. The transport properties of these 2 phases has been described with reference to the carrier drift, or hopping conduction mechanisms. Threshold switching has been reviewed in deep detailed, covering both its fundamental mechanism, its steady-state description, and its dynamics, supporting the fundamental role of a detailed electrical characterization for accurate modeling and design of PCM memory circuits.

\section{Acknowledgments}

This work was supported in part by the European Research Council Consolidator Grant ERC-2014-CoG-648635RESCUE.

\section{References}

[1] M. Wuttig and N. Yamada, "Phase-change materials for rewriteable data storage," Nature Materials 6, 824 832 (2007).

[2] H.-S. P. Wong, S. Raoux, S. Kim, J. Liang, J. P. Reifenberg, B. Rajendran, M. Asheghi, and K. E. Goodson, "Phase change memory," Proc. IEEE 98, 2201 (2010).

[3] S. Raoux, W. Welnic and D. Ielmini, "Phase change materials and their application to non-volatile memories," Chem. Rev. 110, 240-267 (2010).

[4] D. Ielmini and A. L. Lacaita, "Phase change materials in non-volatile storage," Materials Today 14, 600-607 (2011).

[5] S. Raoux, D. Ielmini, M. Wuttig and I. V. Karpov, "Phase change materials," MRS Bull. 37, 118-123 (2012).

[6] N. Yamada, E. Ohno, K. Nishiuchi, N. Akahira, and M. Takao, J. Appl. Phys. 69, 2849 (1991).

[7] D. Mantegazza, D. Ielmini, A. Pirovano, A. L. Lacaita, E. Varesi, F. Pellizzer and R. Bez, "Explanation of programming distributions in phase-change memory arrays based on crystallization time statistics," Solid-State Electronics. 52, 584-590 (2008).

[8] F. Pellizzer, A. Pirovano, F. Ottogalli, M. Magistretti, M. Scaravaggi, P. Zuliani, M. Tosi, A. Benvenuti, P. Besana, S. Cadeo, T. Marangon, R. Morandi, R. Piva, A. Spandre, R. Zonca, A. Modelli, E. Varesi, T. Lowrey, A. Lacaita, G. Casagrande, P. Cappelletti, and R. Bez, Symp. VLSI. Tech. Dig., 18 (2004).

[9] F. Pellizzer, A. Benvenuti, B. Gleixner, Y. Kim, B. Johnson, M. Magistretti, T. Marangon, A. Pirovano, R. Bez, G. Atwood, Symp. VLSI Tech. Dig. 122 (2006). 
[10] G. Servalli, IEDM Tech. Dig. 113 (2009).

[11] B.-S. Lee, J. R. Abelson, S. G. Bishop, D.-H. Kang, B.-K. Cheong, and K.-B. Kim, "Investigation of the optical and electronic properties of $\mathrm{Ge}_{2} \mathrm{Sb}_{2} \mathrm{Te}_{5}$ phase change material in its amorphous, cubic, and hexagonal phases,” J. Appl. Phys. 97, 093509 (2005).

[12] T. Ide, M. Suzuki, and M. Okada, Jpn. J. Appl. Phys., Part 2 34, L529 (1995).

[13] D. V. Tsu, J. Vac. Sci. Technol. A 17, 1854 (1999).

[14] S. Y. Kim, S. J. Kim, H. Seo, and M. R. Kim, Jpn. J. Appl. Phys., Part 138, 1713 (1999).

[15] E. García-García, A. Mendoza-Galván, Y. Vorobiev, E. Morales-Sánchez, J. González-Hernández, G. Martínez, and B. S. Chao, J. Vac. Sci. Technol. A 17, 1805 (1999).

[16] R. Liang, C. Peng, K. Nagata, K. Daly-Flynn, and M. Mansuripur, Appl. Opt. 41, 370 (2002).

[17] H. B. Yao, L. P. Shi, T. C. Chong, P. K. Tan, and X. S. Miao, Jpn. J. Appl. Phys., Part 1 42, 828 (2003).

[18] S. H. An, X. Li, and S. Y. Kim, Jpn. J. Appl. Phys., Part 143, 1006 (2004).

[19] A. Pirovano, A. L. Lacaita, A. Benvenuti, F. Pellizzer, and R. Bez, IEEE Trans. Electron Devices 51, 452 (2004).

[20] N. F. Mott and E. A. Davis, Electronic Processes in Non-Crystalline Materials, 2nd ed. (Clarendon, Oxford, New York, 1979).

[21] J. Tauc, R. Grigorovici, and A. Vancu, Phys. Status Solidi 15, 627 (1966).

[22] T. Kato and K. Tanaka, "Electronic properties of amorphous and crystalline $\mathrm{Ge}_{2} \mathrm{Sb}_{2} \mathrm{Te}_{5}$ films," Jpn. J. Appl. Phys. 44, 7340-7344 (2005).

[23] J.-J. Kim, K. Kobayashi, E. Ikenaga, M. Kobata, S. Ueda, T. Matsunaga, K. Kifune, R. Kojima, and N. Yamada, "Electronic structure of amorphous and crystalline (GeTe)1-x(Sb2Te3)x investigated using hard x-ray photoemission spectroscopy," Phys. Rev. B 76, 115124 (2007).

[24] N. Yamada and T. Matsunaga, "Structure of laser-crystallized Ge Sb Te sputtered thin films for use in optical memory,” J. Appl. Phys. 88, 7020-7028 (2000).

[25] T. H. Lee, and S. R. Elliott, "Structural role of vacancies in the phase transition of Ge2Sb2Te5 memory materials," Phys. Rev. B 84, 094124 (2011).

[26] L. E. Shelimova, O. G. Karpinskii, P. P. Konstantinov, M. A. Kretova, E. S. Avilov, and V. S. Zemskov, Inorg. Mater. 37, 342 (2001).

[27] D. Ielmini, D. Sharma, S. Lavizzari and A. L. Lacaita, "Physical mechanism and temperature acceleration of relaxation effects in phase change memory cells," IEEE International Reliability Physics Symposium (IRPS), 597-603 (2008).

[28] M. H. Cohen, H. Fritzsche, and S. R. Ovshinsky, "Simple band model for amorphous semiconducting alloys," Phys. Rev. Lett. 22, 1065-1068 (1969). 
[29] R. A. Street and N. F. Mott, "States in the gap in glassy semiconductors," Phys. Rev. Lett. 35, 1293 (1975).

[30] S. R. Ovshinsky, "Localized states in the gap of amorphous semiconductors," Phys. Rev. Lett. 36, 1469$1472(1976)$.

[31] N. Ciocchini, E. Palumbo, M. Borghi, P. Zuliani, R. Annunziata and D. Ielmini, "Unified reliability modeling of Ge-rich phase change memory for embedded applications," IEDM Tech. Dig. 581 (2013).

[32] Y. Sasago, M. Kinoshita, T. Morikawa, K. Kurotsuchi, S. Hanzawa, T. Mine, A. Shima, Y. Fujisaki, H. Kume, H. Moriya, N. Takaura and K. Torii, Symp. VLSI Tech. Dig. 24 (2009).

[33] D. Kau, S. Tang, I. V. Karpov, R. Dodge, B. Klehn, J. A. Kalb, J. Strand, A. Diaz, N. Leung, J. Wu, S. Lee, T. Langtry, K.-W. Chang, C. Papagianni, J. Lee, J. Hirst, S. Erra, E. Flores, N. Righos, H. Castro and G. Spadini, IEDM Tech. Dig. 617 (2009).

[34] G. W. Burr, K. Virwani, R. S. Shenoy, A. Padilla, M. BrightSky, E. A. Joseph, M. Lofaro, A. J. Kellock, R. S. King, K. Nguyen, A. N. Bowers, M. Jurich, C. T. Rettner, B. Jackson, D. S. Bethune, R. M. Shelby, T. Topuria, N. Arellano, P. M. Rice, B. N. Kurdi, and K. Gopalakrishnan, "Large-scale (512kbit) integration of Multilayer-ready Access-Devices based on Mixed-Ionic-Electronic-Conduction (MIEC) at 100\% yield," Symp. VLSI Tech. Dig. 41-42 (2012).

[35] A. Calderoni, M. Ferro, E. Varesi, P. Fantini, M. Rizzi, and D. Ielmini, "Understanding Over-Reset Transition in Phase Change Memory (PCM) Characteristics," IEEE Electron Device Lett. 33, 1267-1269 (2012).

[36] M. Rizzi, M. Ferro, P. Fantini, and D. Ielmini, "Energy Landscape Model of Conduction and Phase Transition in Phase Change Memories," IEEE Trans. Electron Devices 60, 3618-3624 (2013).

[37] Y. H. Shih, M. H. Lee, M. Breitwisch, R. Cheek, J. Y. Wu, B. Rajendran, Y. Zhu, E. K. Lai, C. F. Chen, H. Y. Cheng, A. Schrott, E. Joseph, R. Dasaka, S. Raoux, H. L. Lung, and C. Lam, "Understanding Amorphous States of Phase-Change Memory Using Frenkel-Poole Model,” IEDM Tech. Dig. 753-756 (2009).

[38] M. Le Gallo, A. Athmanathan, D. Krebs, and A. Sebastian, "Evidence for thermally assisted threshold switching behavior in nanoscale phase-change memory cells,” J. Appl. Phys. 119, 025704 (2016).

[39] N. Ciocchini, M. Laudato, M. Boniardi, E. Varesi, P. Fantini, A. L. Lacaita, and D. Ielmini, "Bipolar switching in chalcogenide phase change memory," Sci. Rep. 6, 29162 (2016).

[40] P. Fantini, S. Brazzelli, E. Cazzini, and A. Mani, "Band gap widening with time induced by structural relaxation in amorphous $\mathrm{Ge}_{2} \mathrm{Sb}_{2} \mathrm{Te}_{5}$ films," Appl. Phys. Lett. 100, 013505 (2012).

[41] S. R. Ovshinsky, "Reversible Electrical Switching Phenomena in Disordered Structures," Phys. Rev. Lett. 21,1450 (1968).

[42] H. Fritzsche, "Physics of instabilities in amorphous semiconductors," IBM J. Res. Develop. 13, 515-521 (1969).

[43] A. E. Owen and J. M. Robertson, "Electronic conduction and switching in chalcogenide glasses," IEEE Trans. Electron Devices 20, 105-122 (1971). 
[44] A. C. Warren, "Reversible thermal breakdown as a switching mechanism in chalcogenide glasses," IEEE Trans. Electron Devices 20, 123-130 (1973).

[45] D. Adler, M. S. Shur, M. Silver, and S. R. Ovshinsky, "Threshold switching in chalcogenide- glass thin films,” J. Appl. Phys. 51, 3289-3309 (1980).

[46] D. Emin, "Current-driven threshold switching of a small polaron semiconductor to a metastable conductor," Phys. Rev. B 74, 035206 (2006).

[47] D. Ielmini and Y. Zhang, "Analytical model for subthreshold conduction and threshold switching in chalcogenide-based memory devices,” J. Appl. Phys. 102, 054517 (2007).

[48] D. Ielmini, "Threshold switching mechanism by high-field energy gain in the hopping transport of chalcogenide glasses," Phys. Rev. B 78, 035308 (2008).

[49] V. G. Karpov, Y. A. Kryukov, I. V. Karpov, and M. Mitra, "Field-induced nucleation in phase change memory," Phys. Rev. B 78, 052201 (2008).

[50] S. Hudgens, "Progress in understanding the Ovshinsky effect: Threshold switching in chalcogenide amorphous semiconductors," physica status solidi B 249, 1951-1955 (2012).

[51] G. W. Burr, Pi. Tchoulfian, T. Topuria, C. Nyffeler, K. Virwani, A. Padilla, R. M. Shelby, M. Eskandari, B. Jackson, and B.-S. Lee, "Observation and modeling of polycrystalline grain formation in $\mathrm{Ge}_{2} \mathrm{Sb}_{2} \mathrm{Te}_{5}$," J. Appl. Phys. 111, 104308 (2012).

[52] N. Ciocchini, E. Palumbo, M. Borghi, P. Zuliani, R. Annunziata, D. Ielmini, "Modeling resistance instabilities of set and reset states in phase change memory with Ge-rich GeSbTe," IEEE Trans. Electron Devices 61, 2136-2144 (2014).

[53] T. Siegrist, P. Jost, H. Volker, M. Woda, P. Merkelbach, C. Schlockermann and M. Wuttig, "Disorderinduced localization in crystalline phase-change materials," Nature Mater. 10, 202-208 (2011).

[54] D. Ielmini, D. Sharma, S. Lavizzari and A. L. Lacaita, "Reliability impact of chalcogenide-structure relaxation in phase change memory (PCM) cells - Part I: Experimental study," IEEE Trans. Electron Devices 56, 1070-1077 (2009).

[55] N. Ciocchini, M. Cassinerio, D. Fugazza and D. Ielmini, "Modeling of threshold voltage drift in phase change memory (PCM) devices,” IEEE Trans. Electron Devices 59, 3084-3090 (2012).

[56] D. Ielmini and Y. Zhang, "Evidence for trap-limited transport in the subthreshold conduction regime of chalcogenide glasses," Appl. Phys. Lett. 90, 192102 (2007).

[57] D. Fugazza, D. Ielmini, S. Lavizzari and A. L. Lacaita, "Distributed-Poole-Frenkel modeling of anomalous resistance scaling and fluctuations in phase-change memory (PCM) devices," IEDM Tech. Dig. 723-726 (2009).

[58] S. C. Agarwal, "Role of potential fluctuations in phase-change GST memory devices," physica status solidi B 249, 1956-1961 (2012)

[59] A. K. Jonscher, J. Phys. C 4, 1331 (1971). 
[60] A. K. Jonscher and C. K. Loh, J. Phys. C 4, 1341 (1971).

[61] A. K. Jonscher and R. M. Hill, in Electrical Conduction in Disordered Nonmetallic Films, Physics of Thin Films, edited by G. Hass, M. H. Francombe, and R. W. Hoffman (Academic, New York, 1975), Vol. 8.

[62] S. Tam, P.-K. Ko and C. Hu, "Lucky-electron model of channel hot-electron injection in MOSFET'S," IEEE Trans. Electron Devices 31, 1116-1125 (1984).

[63] S. Lavizzari, D. Ielmini and A. L. Lacaita, "Transient simulation of delay and switching effects in phase change memories," IEEE Trans. Electron Devices 57, 3257-3264 (2010).

[64] D. Ielmini, D. Mantegazza, A. L. Lacaita, A. Pirovano and F. Pellizzer, "Parasitic reset in the programming transient of phase change memories," IEEE Electron Device Lett. 26, 799-801 (2005).

[65] S. Lavizzari, D. Sharma and D. Ielmini, "Threshold-switching delay controlled by $1 /$ f current fluctuations in phase-change memory devices," IEEE Trans. Electron Devices 57, 1047-1054 (2010).

[66] S. Lavizzari, D. Ielmini, D. Sharma and A. L. Lacaita, "Transient effects of delay, switching and recovery in phase change memory (PCM) devices,” IEDM Tech. Dig., 215-218 (2008).

[67] M. Cassinerio, N. Ciocchini, and D. Ielmini, "Logic computation in phase change materials by threshold and memory switching," Adv. Mater. 25, 5975-5980 (2013).

[68] D. Loke, J. M. Skelton, W.-J. Wang, T.-H. Lee, R. Zhao, T.-C. Chong, and S. R. Elliott, "Ultrafast phasechange logic device driven by melting processes," Proc. Nat. Academ. Sci. 111, 13272-13277 (2014).

[69] Y. Li, Y. P. Zhong, Y. F. Deng, Y. X. Zhou, L. Xu and X. S. Miao, "Nonvolatile "AND," "OR,” and "NOT" Boolean logic gates based on phase-change memory," J. Appl. Phys. 114, 234503 (2013).

[70] D. Kuzum, R. G. D. Jeyasingh, B. Lee, and H.-S. P. Wong, "Nanoelectronic programmable synapses based on phase change materials for brain-inspired computing. NanoLett. 12, 2179 (2012).

[71] G. W. Burr, R. M. Shelby, S. Sidler, C. di Nolfo, J. Jang, I. Boybat, R. S. Shenoy, P. Narayanan, K. Virwani, E. U. Giacometti, B. N. Kurdi, and H. Hwang, "Experimental Demonstration and Tolerancing of a Large-Scale Neural Network (165 000 Synapses) Using Phase-Change Memory as the Synaptic Weight Element," IEEE Trans. Electron Devices 62, 3498 (2015).

[72] S. Ambrogio, N. Ciocchini, M. Laudato, V. Milo, A. Pirovano, P. Fantini and D. Ielmini, "Unsupervised learning by spike timing dependent plasticity in phase change memory (PCM) synapses," Front. Neurosci. 10:56 (2016).

[73] D. Ielmini, S. Lavizzari, D. Sharma and A. L. Lacaita "Physical interpretation, modeling and impact on phase change memory (PCM) reliability of resistance drift due to chalcogenide structural relaxation," IEDM Tech. Dig., 939-942, (2007).

[74] A. Pirovano, A. L. Lacaita, F. Pellizzer, S. A. Kostylev, A. Benvenuti, and R. Bez, "Low-Field Amorphous State Resistance and Threshold Voltage Drift in Chalcogenide Materials," IEEE Trans. Electron Devices 51, 714 (2004). 
[75] D. Ielmini, A. L. Lacaita, and D. Mantegazza, "Recovery and drift dynamics of resistance and threshold voltages in phase change memories," IEEE Trans. Electron Devices 54, 308 (2007).

[76] S. Gabardi, S. Caravati, G. C. Sosso, J. Behler, and M. Bernasconi, "Microscopic origin of resistance drift in the amorphous state of the phase-change compound GeTe," Phys. Rev. B 92, 054201 (2015).

[77] S. Roorda, W. C. Sinke, J. M. Poate, D. C. Jacobson, S. Dierker, B. S. Dennis, D. J. Eaglesham, F. Spaepen, and P. Fuoss, "Structural relaxation and defect annihilation in pure amorphous silicon," Phys. Rev. B, Condens. Matter, vol. 44, no. 8, pp. 3702-3725, Aug. 1991.

[78] K. P. Chik, S. Y. Feng, and S. K. Poon, "Generation of dangling bonds by high temperature annealing and hopping conduction in amorphous silicon films," Solid State Commun., vol. 33, no. 10, pp. 1019-1023, Mar. 1980.

[79] E. P. Donovan, F. Spaepen, D. Turnbull, J. M. Poate, and D. C. Jacobson, "Calorimetric studies of crystallization and relaxation of amorphous Si and Ge prepared by ion implantation,” J. Appl. Phys., vol. 57, no. 6, pp. 1795-1804, Mar. 1985.

[80] L. L. Snead and S. J. Zinkle, "Structural relaxation in amorphous silicon carbide," Nucl. Instrum. Methods Phys. Res. B, Beam Interact. Mater. At., vol. 191, no. 1-4, pp. 497-503, May 2002.

[81] N. M. J. Conway, A. Ilie, J. Robertson, W. I. Milne, and A. Tagliaferro, "Reduction in defect density by annealing in hydrogenated tetrahedral amorphous carbon,” Appl. Phys. Lett., vol. 73, no. 17, pp. 2456-2458, Oct. 1998.

[82] M. Boniardi and D. Ielmini, "Physical origin of the resistance drift exponent in amorphous phase change materials,” Appl. Phys. Lett. 98, 243506 (2011).

[83] M. Boniardi, D. Ielmini, S. Lavizzari, A. L. Lacaita, A. Redaelli and A. Pirovano, "Statistics of resistance drift due to structural relaxation in phase-change memory arrays," IEEE Trans. Electron Devices 57, 2690-2696 (2010).

[84] A. Sebastian, N. Papandreou, A. Pantazi, H. Pozidis, and E. Eleftheriou, "Non-resistance-based cell-state metric for phase-change memory," J. Appl. Phys., vol. 110, no. 08, p. 084505 (2011).

[85] S. Kim, N. Sosa, M. BrightSky, D. Mori, W. Kim, Y. Zhu, K. Suu, and C. Lam, "A Phase Change Memory Cell with Metallic Surfactant Layer as a Resistance Drift Stabilizer," IEDM Tech. Dig. 762 (2013). 